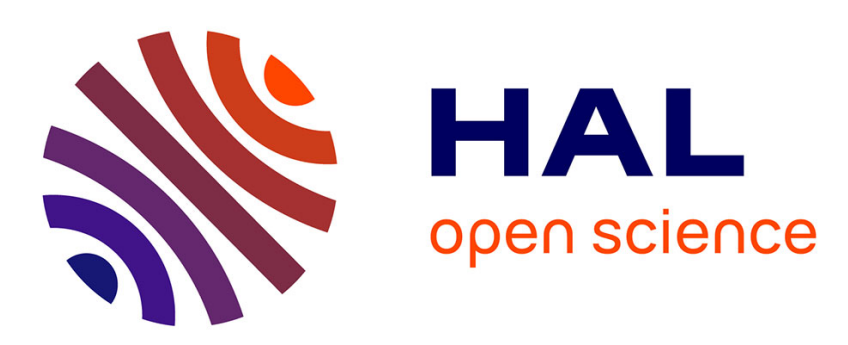

\title{
The p53 protein and its molecular network: modelling a missing link between DNA damage and cell fate
}

Jan Elias, Luna Dimitrio, Jean Clairambault, Roberto Natalini

\section{To cite this version:}

Jan Elias, Luna Dimitrio, Jean Clairambault, Roberto Natalini. The p53 protein and its molecular network: modelling a missing link between DNA damage and cell fate. Biochimica et Biophysica Acta Proteins and Proteomics, 2013, 10.1016/j.bbapap.2013.09.019 . hal-00822308v2

\section{HAL Id: hal-00822308 \\ https://hal.inria.fr/hal-00822308v2}

Submitted on 11 Oct 2013

HAL is a multi-disciplinary open access archive for the deposit and dissemination of scientific research documents, whether they are published or not. The documents may come from teaching and research institutions in France or abroad, or from public or private research centers.
L'archive ouverte pluridisciplinaire HAL, est destinée au dépôt et à la diffusion de documents scientifiques de niveau recherche, publiés ou non, émanant des établissements d'enseignement et de recherche français ou étrangers, des laboratoires publics ou privés. 


\title{
The p53 protein and its molecular network: modelling a missing link between DNA damage and cell fate
}

\author{
Ján Eliaš ${ }^{\mathrm{a}, \mathrm{b}}$, Luna Dimitrio ${ }^{\mathrm{a}, \mathrm{b}, \mathrm{c}}$, Jean Clairambault ${ }^{\mathrm{a}, \mathrm{b}}$, Roberto Natalini ${ }^{\mathrm{d}, \mathrm{e}}$ \\ ${ }^{a}$ UPMC, Laboratoire Jacques-Louis Lions, 4 Place Jussieu, F-75005 Paris, France \\ ${ }^{b}$ INRIA Paris-Rocquencourt, Bang project-team, Paris and Rocquencourt, France \\ ${ }^{c}$ On leave to SANOFI, Vitry, France \\ ${ }^{d}$ Istituto per le Applicazioni del Calcolo "Mauro Picone", CNR, Rome, Italy \\ e Dipartimento di Matematica, Università di Roma "Tor Vergata", Rome, Italy
}

\begin{abstract}
Various molecular pharmacokinetic-pharmacodynamic (PK-PD) models have been proposed in the last decades to represent and predict drug effects in anticancer chemotherapies. Most of these models are cell population based since clearly measurable effects of drugs can be seen, much more easily than in individual cells, on populations of cells, healthy and tumour.

The actual targets of drugs are, however, cells themselves. The drugs in use either disrupt genome integrity by causing DNA strand breaks, and consequently initiate programmed cell death, or block cell proliferation mainly by inhibiting factors that enable cells to proceed from one cell cycle phase to the next through checkpoints in the cell division cycle. DNA damage caused by cytotoxic drugs (and also cytostatic drugs at high concentrations) activates, among others, the p53 protein-modulated signalling pathways that directly or indirectly force the cell to make a decision between survival and death.

The paper aims to become the first-step in a larger scale enterprise that should bridge the gap between intracellular and population PK-PD models, providing oncologists with a rationale to predict and optimise the effects of anticancer drugs in the clinic. So far, it only sticks at describing p53 activation and regulation in single cells following their exposure to DNA damaging stress agents. We show that p53 oscillations that have been observed in individual cells can be reconstructed and predicted by compartmentalising cellular events occurring after DNA damage, either in the nucleus or in the cytoplasm, and by describing network interactions, using ordinary differential equations (ODEs), between the ATM, p53, Mdm2 and Wip1 proteins, in each compartment, nucleus or cytoplasm, and between the two compartments.
\end{abstract}




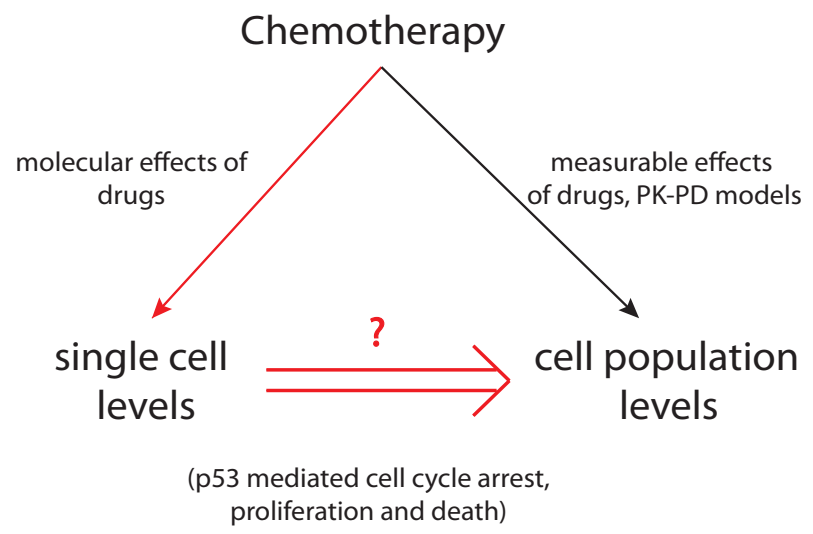

Keywords: p53, DNA damage, ATM, cell fate, molecular mathematical model

\section{Introduction}

Shortly after disruption of the integrity of the genome of a cell by various pharmacological agents or ionising radiations, the cell responds dynamically by activating a variety of recognition and repair proteins recruited to DNA damage sites, by initiating various signalling pathways leading either to cell cycle arrest and parallel DNA repair, or permanent cell cycle arrest, or else cell death. Among these pathways, one of the most important ones is the network of the tumour suppressor protein $\mathrm{p} 53$, the so-called guardian of the genome, that initiates expression of those genes that ultimately govern cell cycle arrest, DNA damage repair and apoptosis, involving the production of proteins of concentrations proportionally related to the concentrations of p53 [1]. At the cell population level (i.e., tissues, organs, whole human body) pharmacokinetics-pharmacodynamics (PKPD) modelling has been broadly used to fully describe absorption, distribution, metabolism, excretion and toxicity of anticancer drugs. Much less, however, has been done at the single cell (molecular) level to describe drug effects, considering that individual cells are the actual targets of drug administration [2]. Some drugs (e.g., the cytotoxic drug, alkylating agent, oxaliplatin) directly cause DNA double strand breaks (DSBs), others target essential cell cycle enzymes (such as topoisomerases or thymidylate synthase), leading to the production of abnormal DNA and forcing the cell to start the process of apoptosis, at least when DNA cannot be repaired [3].

Thus, to reproduce more realistically drug effects in cancer treatments, as described by PK-PD models with DNA damage as output, it may be helpful to 
include in existing models processes that appear in individual cells after DNA insult, beginning with a proper understanding of p53 activation and activity in single cells, with the perspective of subsequent integration of such activity into a cell fate decision process. Bearing in mind that p53 is inactive due to its gene mutations in around $50 \%$ of tumour cells, with the rate varying from 10-12\% in leukaemia, $38-70 \%$ in lung cancers to $43-60 \%$ in colon cancers, etc. [4], approaches involving processes occurring in individual cells with the dominant role of p53 can contribute to establishing new cancer therapies that could either restore p53 lost functionality or substitute for it in the activation of subsequent proteins in various p53-initiated pathways.

In this modelling enterprise, the main object of interest at the single cell level is thus the protein p53, its activation and its activity on proarrest and proapoptotic genes that enable the cell to make a decision between cell cycle arrest and DNA repair, permanent arrest of cell growth (so-called senescence) and cell death (apoptosis). Note that the first p53-transcription independent wave of cells committing apoptosis in response to $\gamma$-irradiation is observed $30 \mathrm{~min}$ after DNA damage by rapid accumulation of p53 in the mitochondria. The second wave comes after a longer time phase and a decision of the cell to undergo apoptosis in this wave is determined by the concentrations of both proapoptotic and proarrest proteins, the expression of which is modulated by p53 [1, 4]. However, different sorts of such apoptotic proteins are produced, in a cell-stress, cell-type and tissue-type dependent manner. Various post-translational modifications, interactions of p53 with over 100 cellular cofactors and p53 cellular location have effects on determining what kind of proteins and when these proteins are produced [4].

The regulation of p53 is mainly achieved through its interactions with the Mdm2 ligase, that itself is a transcription target for $\mathrm{p} 53$. Mdm2 regulates $\mathrm{p} 53$ through (multiple-)ubiquitination process, followed by nuclear export and subsequent degradation of tagged p53 molecules. Phosphorylation of p53 serine 15 (Ser15) residue, which is located very close to the p53 kinase domain — the target of Mdm2 - can mask it from Mdm2 ubiquitination and hence stabilise it at its highest concentrations $[5,6,7]$. In response to DSBs, p53 can be phosphorylated on Ser15 in three independent ways, among which is phosphorylation by the ATM kinase [7]. Phosphatase Wip1 is another p53 target which acts in the pathway as a regulator; particularly, it dephosphorylates both ATM and p53, rendering them inactive, whence Wip1 closes negative feedback loops between these proteins as is schematically shown on Figure 1.1.

In this article we model and simulate in silico ATM/p53/Mdm2/Wip1 dynamics of protein interactions in the p53 signalling pathway in response to DNA DSBs 


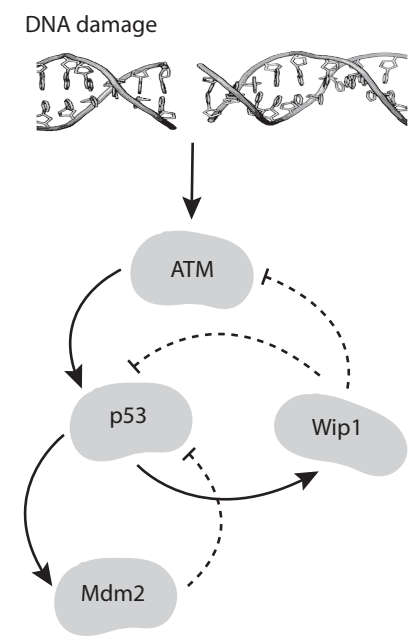

Figure 1.1: The ATM/p53/Mdm2/Wip1 dynamics.

at the single cell level, aiming at further representing cell fate decision in future cell population models. This model is indeed intended to become a first step in narrowing the gap between intracellular and cell population PK-PD models. Compartmental and spatial p53/Mdm2 models developed by Dimitrio et al. $[5,6]$ are here extended by considering the ATM and Wip1 proteins. In these models ATM is interpreted as an identifier of DNA DSBs, although it is not a direct DNA DSB sensor [8]. However, unlike in the originally proposed model, and with the perspective to involve 553 in further PK-PD models, we integrate ATM and Wip1 into the p53/Mdm2 dynamics so that p53 activation and regulation can be modelled more plausibly with respect to relevant biological observations. In the original model, compartmentalisation of cellular events together with $\mathrm{p} 53 / \mathrm{Mdm} 2$ feedback ( $p 53 \rightarrow M d m 2 \dashv 53$ ) led to the desired p53 oscillatory behaviour even with constant ATM parameter (considered as a measure of DNA damage). Under our new assumptions, ATM concentration is considered as a continuous timedependent function, and we show that the feedbacks $p 53 \rightarrow M d m 2 \dashv p 53$ and $A T M \rightarrow p 53 \rightarrow$ Wip $1 \dashv A T M$ together with compartmental distribution of proteins can then reconstruct the oscillatory dynamics of these proteins.

The paper is organised as follows: In Section 2, we give a short biological background of our subject in relation with physiologically based PK-PD modelling. Then in Section 3, we present motivations for our modelling work, by firstly describing in detail known mechanisms of p53 activation, p53 activity towards its substrates and links between DNA damage and ATM activation. Then 
specifications for the proposed model and its computer simulation are presented in Section 4; this section contains our results with their physiological interpretations. Mathematical details, including the complete systems of equations with the parameters chosen for simulations, and some illustrative plots, are presented in the Appendices A to D.

\section{Cancer therapies and PK-PD models}

Most cytotoxic and cytostatic drugs used in cancer treatments act in a selective manner, taking advantage of differences in tumour cell characteristics, compared to healthy cells, such as high proliferation rates, genome instability and tolerance to hypoxia. This results in proper targeting of chemotherapeutic agents on tumour cells. However, cancer therapies also have toxic side effects on healthy cells and can lead to the disruption of physiological functionality of tissues and organs. In the particular case of chronotherapeutic settings (i.e., hypothesizing best treatment periods in the 24 hour span), physiologically based molecular PK-PD models using ODEs have been proposed for the cytotoxic drugs Oxaliplatin [3], 5-Fluorouracil [3,9] and Irinotecan [10, 11], all three drugs that are commonly used in the treatment of metastatic colorectal cancer. Moreover, to minimise such unwanted effects in more general (non chrotherapeutic) settings, most anticancer drugs are delivered in the clinic in time interval-depending regimens to allow cells to recover their normal functions after pharmacological insult. However, time intervals between chemotherapy courses also allow tumour cells to recover, activating their survival mechanisms, and further resist subsequent therapeutic insults. Optimal scheduling strategies are currently being theoretically investigated $[2,3,12]$ to obtain satisfying trade-offs between the objective of reducing the tumour burden without inducing neither intolerable side effects on healthy cells nor emergence of resistant clones in the tumour cell population.

Besides toxic side effects on healthy cells, tumour cells can indeed often develop multiple resistance to drugs. Moreover, in vivo observations in the tumour stromal tissue surrounding tumours of prostate, breast and ovary reveal the druginduced production of a spectrum of secreted cytokines and growth factors, e.g., WNT16B, that promote tumour growth and survival of cancer cells after cytotoxic therapy, and further reduce chemotherapy sensitivity in tumour cells, resulting in tumour progression; otherwise said, among other constraints, one must take into account the fact that chemotherapy itself can positively influence the growth of tumours [13]. 
In order to take into account these different therapy-limiting constraints, optimal administration of anticancer agents should involve accurately representing the action of drugs at the molecular, i.e., intracellular, level, which means molecular pharmacokinetic-pharmacodynamic (PK-PD) modelling for each drug used in a given treatment firstly at the level of a single cell and secondly (not described in this work) at the level of cell populations, i.e., tissues and organs. The review we present here of p53 molecular mechanisms of action, and their modelling, is positioned in continuity with, but downstream of, the action of cytotoxic anticancer drugs, when DNA damage is constituted, so that DNA damage is the interface between molecular PK-PD models (not recalled here) and the model we propose in Section 4, based on physiological mechanisms reviewed in Section 3.

\section{3. p53 signalling in DNA damage response}

Currently, one can find in the scientific literature a large number of papers contributing to describing p53 signalling in detail. In the following sections we point out only the most important facts that are further recalled and used in the model development. Detailed overviews of the p53 transcriptional activity can be found in the works of Murray-Zmijewski et al. [4] and Vogelstein et al. [7].

\section{1. p53 activation and regulation}

The tumour suppressor protein p53 can be activated in at least three independent ways: DNA damage caused, for example, by ionising radiation or electromagnetic $\gamma$ radiation, with initial activation of ATM and Chk2 proteins; aberrant growth signals; and various chemotherapeutic drugs, UV radiation and proteinkinase inhibitors. All three ways inhibit p53 degradation and enable the protein to accomplish its main transcriptional function [7].

The concentration of p53 in cells is determined mainly through its degradation. To prevent p53 degradation following DNA damage, ATM (or Chk1, Chk2 and DNA-dependent protein kinases) phosphorylates p53 on Ser15 localised at the amino-terminal sites (in vitro and in vivo) very close to the binding site of its main regulator Mdm2. Phosphorylation of Ser15 masks p53 from Mdm2 (it blocks binding Mdm2 to p53); it stabilises p53 at high concentrations and thus it initiates p53 transcriptional activity $[5,6,7]$.

Phosphatase Wip1, a transcription target of p53, is then observed to act in the reverse way, compared with the action of ATM. It dephosphorylates both ATM and p53, making them inactive and unable to phosphorylate their substrates; in 
particular, inactive ATM cannot phosphorylate p53 on Ser15 and dephosphorylated p53 is then detectable by Mdm2, as represented on Figure 1.1 [14, 15, 16].

The E3 ligase Mdm2 is another transcription target of p53. Its p53-inhibiting activity consists in multiple ubiquitination. More precisely, Mdm2 binds the amino-terminus of p53 after Ser15 dephosphorylation and recruits E2 ligases, which directly attach ubiquitines (small peptides) to Lys residues at the carboxylterminus of $\mathrm{p} 53$. The ubiquitinated $\mathrm{p} 53$ protein is then exported to the cytoplasm where it is easily detected by the protein-degrading machinery [7]. Other proteins such as HAUSP can contribute to p53 stability by deubiquitination of p53, i.e. by opposing Mdm2 [4].

Among other things, full p53 transcriptional activation and stabilisation in highly specific situations require other post-translational modifications (phosphorylation, acetylation, methylation, sumoylation, ubiquitination, etc.) of one or more p53 residues. The particular p53 activity is even more complicated considering that different modifications of the same p53 residue, e.g. methylation and ubiquitination of Lys370, result in different p53 effects [4].

\section{2. p53 transcriptional activity towards proarrest and proapoptotic proteins}

The protein p53 as a transcription factor can activate hundreds of genes in response to a variety of stress signals, thus transforming such signals into various cellular responses. In addition, the p53 transcriptional activity is heterogeneous, depending not only on the incoming signal (its type and amplitude) but also on many other factors - the environment of the cell, type of cells, tissues, presence and abundance of cellular cofactors and enzymes causing modifications of over thirty residues of p53, etc.. All this can induce alterations in p53 stability, expression of substrate genes and cellular location [4].

Experiments on p53 activity on its substrates initially suggested that p53 activates genes likely with respect to its affinity for a specific promoter. Such conception, for example, assumed that low concentrations of p53 predominantly lead to the activation of genes of high binding affinity, mostly the genes coding for proarrest proteins. When the concentration of p53 is high, then it activates also genes of low binding affinity (i.e. proapoptotic genes). However, this view has been partially disproved by observations evidencing that post-translational modifications contribute to both proarrest and proapoptotic proteins activation without any preference being due to p53 affinity towards promoters [4]. Recent experiments by Kracikova et al. [1] support these ideas and contradict models based on a differential p53 affinity by showing that even low levels of p53 can activate both 
proarrest and proapoptotic genes, and that concentrations and durations of the expression of p53 substrates are determined only by concentration and duration of expression of p53 itself.

Importantly, a stressed cell evaluates the presence of both proarrest and proapoptotic proteins produced in a p53-dependent manner at any time during its response to DNA damage. The cell determines a so-called "apoptotic ratio" with respect to protein concentrations, duration of their expression and other factors. Irreversible apoptosis launching is initiated whenever this ratio crosses a given threshold [1]. Expression of those apoptosis-launching proteins is a rather complex process that depends on many factors (post-translational modifications, interactions with other cellular substrates, location, etc.), but from an intracellular PK-PD modelling point of view, the most interesting part, i.e. apoptotic response to therapeutic drugs, must certainly involve p53. However, modelling p53-modulated further cell fate decision (apoptosis or repair and survival, with possible senescence) is omitted from this paper and left for further research, simply because of so far insufficient biological knowledge of the intracellular (and intercellular) mechanisms at stake. In this paper, we review, model and simulate the activation and regulation of p53 through the ATM, Wip1 and Mdm2 proteins.

\section{3. p53 oscillations in single cells; dependency of such oscillations on ATM}

Experiments in individual living breast cancer cells show that concentrations of p53 and Mdm2 exhibit sustained oscillations [17] of duration over several days, with slightly varying period and widely varying amplitude from peak to peak, also with a number of pulses different from cell to cell, following $\gamma$-irradiation [18]. Originally observed damped oscillations that have been measured in immunoblots [19] are likely caused by cancelling of pulses in a population of cells. Note also that not all cells exhibit oscillations in proteins after DNA damage; however, the fraction of oscillating cells increases with the irradiation dose [18].

Further single-cell experiments on the p53/Mdm2 dynamics reveal that the p53/Mdm2 feedback loop itself is not sufficient to produce sustained oscillations [14]. Instead, the p53 pulses depend on oscillations of the proteins that sense and transmit the damage signal to p53, particularly ATM and Chk2 (Chk2 itself is a target for ATM), and on the second negative feedback loop between p53 and the phosphatase Wip1, which is schematically illustrated on Figure 1.1. Even thus, the initial activation of p53 by ATM and Chk2 is not sufficient to generate multiple p53 pulses, and sustained p53 pulses are detectable in parallel to sustained ATM and Chk2 oscillations only; whenever ATM activity is inhibited, oscillatory behaviour of p53 also vanishes [14]. 
Hence, although Mdm2 is important for the regulation of $\mathrm{p} 53$, Mdm2 without ATM and Wip1 does not lead to 553 oscillations. We thus propose that a model capturing initiation and regulation of p53 activity must involve the ATM and Wip 1 proteins, the dynamics of which should be fully described by mathematical variables, solutions of ODEs, rather than represented by constant parameters.

\subsection{Compartmentalisation}

The $\mathrm{p} 53$ protein is a predominantly nuclear protein, which is understandable since its main role consists in the transcriptional activation of many other proteins. However, p53 can also play a part outside of the nucleus, notably as a transcription-independent factor involved in apoptosis induction $[4,20]$. Note that there are many cellular substrates affecting p53 location. For example, the E3 ligase E4F1 contributes to stronger association of p53 with chromatin through multiple ubiquitination, and the PARP1 polymerase positively affects nuclear accumulation and inhibits nuclear export. On the other hand, Mdm2-monoubiquitination of p53 can promote p53 sumoylation with the consequences of effective p53 nuclear export [4].

ATM is also predominantly concentrated in the nucleus with only $10-20 \%$ of its molecules found in the cytoplasm, particularly bound to peroxysomes and endosomes [21]. Fluorescent microscopy techniques have confirmed this spatial distribution of ATM between the nucleus and the cytoplasm. However, only the nuclear fraction of ATM is autophosphorylated in response to DNA damage induced by ionising radiation, and activated ATM proteins form detectable foci that are colocalised with foci of $\gamma \mathrm{H} 2 \mathrm{AX}$, a marker of DNA DSBs. Cytoplasmic ATM can neither autophosphorylate itself nor phosphorylate its substrates [22].

The p53-mediated expression of Mdm2 and Wip1 genes produces in the cytoplasm proteins that can freely migrate between the nucleus and the cytoplasm. However, the protein Wip1 is mostly observed to accumulate in the nucleus following ionising radiation [23].

\subsection{ATM activity on $p 53$}

Although p53 activation by ATM in response to DNA damage is well documented, the precise mechanisms of this activation are less well known. We present in Section 3.5.3 an overview of recent biological observations related to the ATM activation and activity. A schematic representation of the ATM protein is shown on Figure 3.1. 


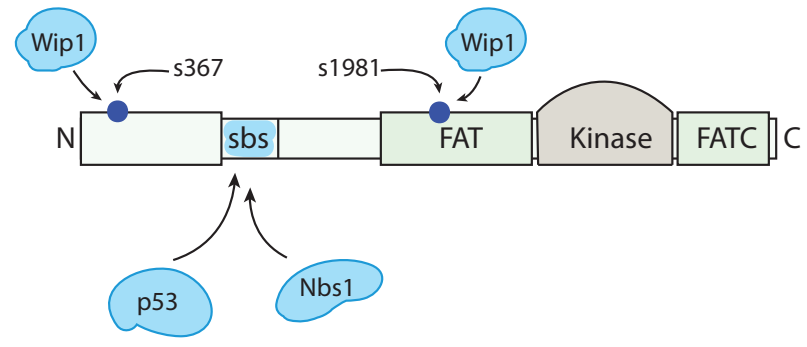

Figure 3.1: Schematic representation of ATM. ATM is a $370 \mathrm{kDa}$ protein with a 350-amino-acid kinase domain between an internal FAT domain, and a carboxylterminus FATC domain. ATM substrates such as p53 and Nbs1 bind to a region near the N-terminus (amino acids 1-989) of the protein substrate binding site (SBS). Deletion of SBS inactivates the protein [21]. The nuclear importins $\alpha 1$ and $\beta 1$ are identified very close to the $\mathrm{N}$-terminus. The $\mathrm{N}$-terminus of ATM is crucial for both nuclear location and chromatin association, optimal ATM activation and subsequent ATM activity in vivo [22]. Wip1 binds to the N-terminus and FAT domain, which contain Ser367 and Ser1981, respectively [16].

\subsubsection{ATM activation}

In unstressed human cells ATM exists in inactive form as a compound formed (dominantly) from two ATM molecules, which makes it stable in cells, retaining it in a constant concentration, and inaccessible to cellular substrates [24, 25]. In this polymer form, the kinase domain of ATM is bound to a region surrounding Ser1981 that is contained within the FAT domain of ATM, as shown on Figure 3.2.

A cell exposure to stress agents (ionising radiation or cytotoxic drugs) induces rapid autophosphorylation of ATM onSer1981 and this phosphorylation results in dimer dissociation and initiation of cellular ATM kinase activity in vivo. In ATM dissociation and activation, the active site of one ATM kinase (one out of two bound in the dimer) catalyses the phosphorylation reaction within which a phosphate group, commonly coming from ATP, is added to Ser1981 of the another ATM kinase (resulting in the so called trans-autophosphorylation), shown on Figure 3.2 [24].

Occasional double strand breaks (DSBs) arising, for instance, from DNA replication are normally promptly corrected by the DNA repair machinery, with either no need to activate ATM or such activation being only moderate and temporary [26]. After DNA damage caused by other agents (at even as low doses as 0.1 Gy of ionising radiation), ATM activation occurs very promptly [24, 27]. ATM forms clearly detectable foci adjacent to DNA DSBs, and even relatively low 

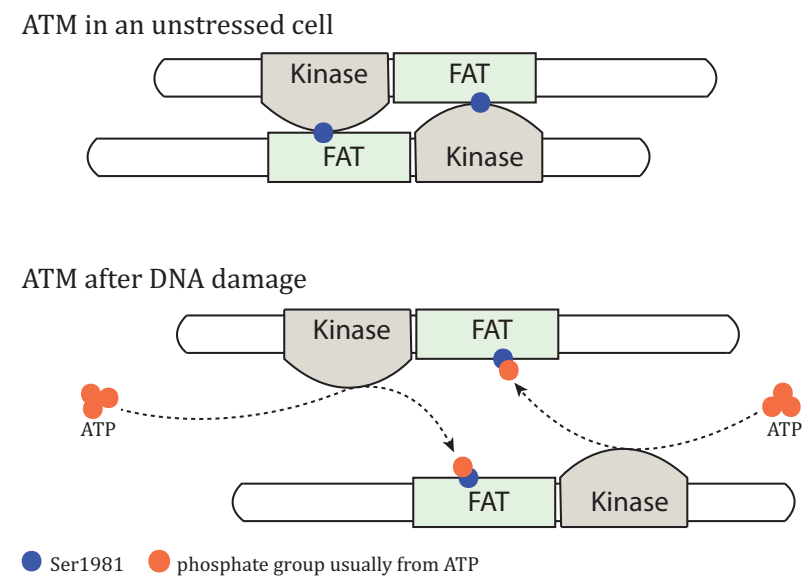

Figure 3.2: ATM forming a dimer in an unstressed cell (above) and dimerisation of the dimer into active monomers through autophosphorylation of Ser1981 after DNA damage.

levels of nuclear ATM may be sufficient to elicit proper responses to DNA damage [22]. In addition to exposure of cells to ionising radiation, cytotoxic drugs and restriction enzymes causing DNA DSBs, phosphorylation of Ser1981 and ATM activation is also detectable by introducing chromatin-modifying treatments such as chloroquine or histone deacetylase inhibitors, which do not induce DSBs [24].

\subsubsection{ATM activity}

In vivo, ATM activation following ionising radiation occurs very rapidly at distance from DNA DSBs, by means of Ser1981 autophosphorylation. Importantly, the kinetics of p53 phosphorylation on Ser15, and thus p53 activation, corresponds to the kinetics of ATM activation. Indeed, phosphorylation of p53 is similarly maximal at doses of $1-3$ Gy of ionising radiation with little or no further increase up to doses of $30 \mathrm{~Gy}$ in fifteen minutes. Clearly detectable p53 phosphorylation also occurs after employing chromatin-modifying treatments not causing DSBs [24, 27].

In contrast, phosphorylation of the ATM substrates SMC1 (on Ser957), Nbs1 (on Ser343) and H2AX (on Ser139) occurs at the sites of DNA DSBs and increases continuously in a dose-dependent manner up to 30 Gy. Furthermore, foci of these proteins are not detectable in case of chromatin modifying treatments [24, 27]. In these cases ATM associates with DNA break sites and the ATM attraction to unwinding DNA break ends is modulated by the C-terminus of 
Nbs1 [28], a part of the complex of Mre11/Rad50/Nbs1 proteins that actually acts as a sensor of DNA DSBs [8].

Similarly to additional post-translational modifications of $\mathrm{p} 53$, ATM contains residues undergoing various modifications, e.g. phosphorylation of the ATM sites Ser367, Ser1893, Ser2996 and Thr1885, that influence various ATM roles in the ATM signalling pathway, mainly at the intra-S phase checkpoint $[29,30]$. Acetylation of Lys3016 by Tip60 has been observed in parallel to Ser1981 phosphorylation as a crucial event in ATM kinase activation and monomerisation [31]. Inactive or phosphorylation site mutants of ATM fail to bind to DNA DSBs in vivo [32].

\subsubsection{ATM regulation}

Unlike for p53, the stability of ATM is not determined through its degradation, but rather by reverse association of monomers to dimers (or multimers), i.e. dephosphorylation of active ATM monomers by some phosphatases and backward dimerisation (multimerisation) of such dephosphorylated monomers.

Abundance and activity of several phosphatases can affect ATM monomerisation and dimerisation, such as phosphatases PP5 [33] and PP2A [34] of the PPP phosphatase family, among which PP2A is likely the only member of this family which can dephosphorylate ATM [34].

Phosphatase Wip1 is also known to dephosphorylate ATM Ser1981 [15]. It has been shown that inefficient Wip1 results in ATM kinase malfunction, and overexpression of Wip1 significantly reduces protein activation in the ATM-dependent signalling cascade after DNA damage. The ATM Ser1981 site is the main target of Wip1 in vivo and in vitro. Moreover, it can dephosphorylate other ATM sites [16]. The protein Wip1 has many other targets in p53 signalling pathway it dephosphorylates, for example, p53, Chk1 and HA2X.

\section{ATM/p53/Mdm2/Wip1 compartmental model}

In this section, we design and analyse a mathematical model that can successfully be used to reconstruct in silico experimentally observed dynamics of proteins, namely, the main cellular actor p53, ATM as a protein transferring the DNA damage signal, Wip1 as a dephosphorylation factor of both p53 and ATM, and Mdm2, that tags p53 for degradation. Note that our model is compartmental in the sense that we strictly distinguish the activities of proteins occurring in the nucleus from those occurring in the cytoplasm, as it is shown on Figure 4.1. Note 
also that the model proposed in this section is an extension of the compartmental model developed by Dimitrio et al. [5, 6].

\subsection{Model specifications}

\subsubsection{Modelling ATM activation and deactivation}

ATM activation, i.e., its autophosphorylation and consequent dissociation of ATM dimers into (active) monomers, can be modelled in several ways. The simplest way is through the dissociation reaction

$$
A T M_{D} \underset{k_{-m}}{\stackrel{k_{m}}{\rightleftharpoons}} A T M_{p}+A T M_{p}
$$

where $A T M_{D}$ denotes a compound of two ATM kinases and $A T M_{p}$ is active (phosphorylated) ATM. In our model, ATM in inactivate state is considered in form of dimers only. The constants $k_{m}$ and $k_{-m}$ denote, respectively, the monomerisation (forward) and dimerisation (reverse) rate constants of reaction. Reaction (1) involves monomerisation (dissociation) of a dimer complex $A T M_{D}$ to the two active ATM species with the rate $k_{m}$ and dimerisation of two $A T M_{p}$ monomers to produce $A T M_{D}$ with the rate $k_{-m}$. The law of mass action then gives mathematical relations between $A T M_{D}$ and $A T M_{p}$ (not shown), with the production of $A T M_{D}$ being equal to the half production of $A T M_{p}$ [35]. A disadvantage of presenting the activation/deactivation of ATM as in (1), however, is that it does not mention any signal initiating $A T M_{D}$ dissociation, whereas such a signal is thought to be produced by changes in chromatin structures after DNA damage [24] and/or by the MRN complex as a DNA strand breaks sensor [8].

Hence, a more convenient way to represent ATM activation with the perspective of further analysis is to involve a hypothetical, likely molecular and so far unidentified DNA damage signal, that will be denoted by $E$, produced by DNA damage recognition sensors, transmitted to and sensed by ATM, resulting in the dissociation of ATM dimers into two molecules of $A T M_{p}$, i.e.,

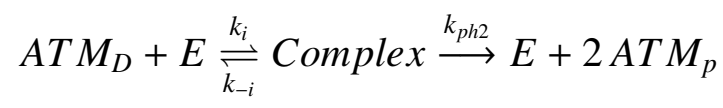

with the corresponding kinetic rates $k_{i}, k_{-i}$ and $k_{p h 2}$. This reaction is not a typical enzymatic reaction since the enzyme-like signal $E$ is in this approach a nonspecified signal corresponding to DNA damage; it is not necessarily an enzyme; however, we assume that $E$ is not amplified, reduced nor otherwise changed in a short time interval. 
ATM deactivation by Wip1 is modelled similarly as

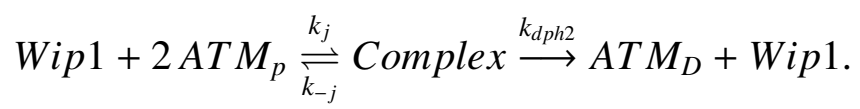

Here, it is assumed that phosphatase Wip1 exists in sufficient concentration to dephosphorylate ATM active monomers in the sense that whenever one $A T M_{p}$ protein is dephosphorylated, another dephosphorylated ATM protein is present, so that they are immediately bound to the dimeric $A T M_{D}$. Kinetic rates are denoted by $k_{j}, k_{-j}$ and $k_{d p h 2}$.

Note that ATM activation and deactivation by Wip1 occur in the nucleus.

The law of mass action and the quasi-steady-state approximation $[35,36]$ then yield differential equations, hereafter (2) and (3); in particular, changes of concentrations in time of $A T M_{D}$ and $A T M_{p}$ are, respectively,

$$
\begin{aligned}
& \frac{d\left[A T M_{D}\right]}{d t}=-k_{p h 2}[E] \frac{\left[A T M_{D}\right]}{K_{p h 2}+\left[A T M_{D}\right]}+k_{d p h 2}[\text { Wip } 1] \frac{\left[A T M_{p}\right]^{2}}{K_{d p h 2}+\left[A T M_{p}\right]^{2}}, \\
& \frac{d\left[A T M_{p}\right]}{d t}=2 k_{p h 2}[E] \frac{\left[A T M_{D}\right]}{K_{p h 2}+\left[A T M_{D}\right]}-2 k_{d p h 2}[\text { Wip } 1] \frac{\left[A T M_{p}\right]^{2}}{K_{d p h 2}+\left[A T M_{p}\right]^{2}},
\end{aligned}
$$

since the production of $A T M_{D}$ is half that of $A T M_{p}$. Here $K_{p h 2}=\frac{k_{-i}+k_{p h 2}}{k_{i}}$ and $K_{d p h 2}=\frac{k_{-j}+k_{d p h 2}}{k_{j}}$ are the Michaelis-Menten rates of reactions (2) and (3), while $k_{p h 2}$ and $k_{d p h 2}$ are the velocities of these reactions, [·] denotes concentration.

ATM has not been observed to be degraded during its signalling activity but, after dephosphorylation by Wip1, it rather forms a compound with another ATM dephosphorylated kinase, thus preserving ATM in a stable concentration [24, 32]. Hence, we require ATM to satisfy a conservation property expressing the fact that the total concentration of nuclear ATM kinases (monomers and kinases bound in dimers) is constant during the considered time period, i.e.,

$$
\left[A T M_{p}\right]+2\left[A T M_{D}\right]=A T M_{T O T},
$$

where $A T M_{T O T}$ is the constant total ATM concentration. Using this conservation property, equations in (4) can actually be merged into one equation. 


\subsubsection{Modelling p53/Mdm2 negative feedback}

The dynamics of p53/Mdm2/Wip1 in the nucleus can be expressed by the following reactions:

$$
\begin{aligned}
& \mathrm{p} 53+M d m 2 \underset{k-}{\stackrel{k}{\rightleftharpoons}} \text { Complex } \stackrel{k_{1}}{\longrightarrow} p 53_{U}+M d m 2 \\
& \text {... for p53 ubiquitination by } \mathrm{Mdm} 2 \text {, }
\end{aligned}
$$

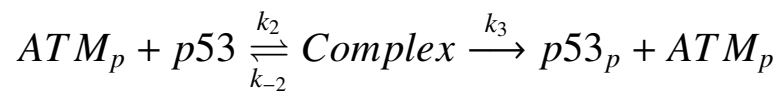

$$
\begin{aligned}
& \text {... for p53 phosphorylation by ATM, }
\end{aligned}
$$

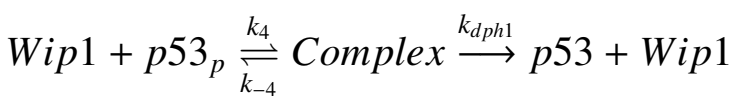

$$
\begin{aligned}
& \text {... for p53 dephosphorylation by Wip1, }
\end{aligned}
$$

with the corresponding kinetic constants.

Again, the application of the law of mass action and the quasi-steady-state approximation yields the equations for time changes of the nuclear inactive $p 53$ and active $p 53_{p}$ concentrations, i.e.,

$$
\begin{aligned}
\frac{d[p 53]}{d t} & =\underbrace{k_{d p h 1}[W i p 1] \frac{\left[p 53_{p}\right]}{K_{d p h 1}+\left[p 53_{p}\right]}}_{\text {dephosphorylation of } p 53_{p} \text { by Wip } 1}-\underbrace{k_{1}[M d m 2] \frac{[p 53]}{K_{1}+[p 53]}}_{\text {ubiquitination of } p 53 \text { by Mdm2 }} \\
& \underbrace{k_{3}\left[A T M_{p}\right] \frac{[p 53]}{K_{a t m}+[p 53]}}_{\text {phosphorylation of } p 53 \text { by } A T M_{p}} \\
\frac{d\left[p 53_{p}\right]}{d t}= & \underbrace{k_{3}\left[A T M_{p}\right] \frac{[p 53]}{K_{a t m}+[p 53]}}_{\text {phosphorylation of } p 53 \text { by } A T M_{p}}-\underbrace{k_{d p h 1}[W i p 1] \frac{\left[p 53_{p}\right]}{K_{d p h 1}+\left[p 53_{p}\right]}}_{\text {dephosphorylation of } p 53_{p} \text { by Wip1 }} .
\end{aligned}
$$

We do not consider p53 gene expression and its mRNA, since, as it has already been mentioned, the principal means of control on p53 concentration by the cell is through the degradation of the protein, dominantly through the multiple Mdm2dependent ubiquitination [4]. For simplicity, p53 degradation is rather modelled as an enzymatic reaction and the ubiquitination of p53 in Equation (6) is thus interpreted as a loss of mass. Note that although p53 degradation controlled by Mdm2 is a preferential way of p53 degradation reported in cells, natural (dominantly cytoplasmic) degradation also occurs, however, with no significant contribution to 


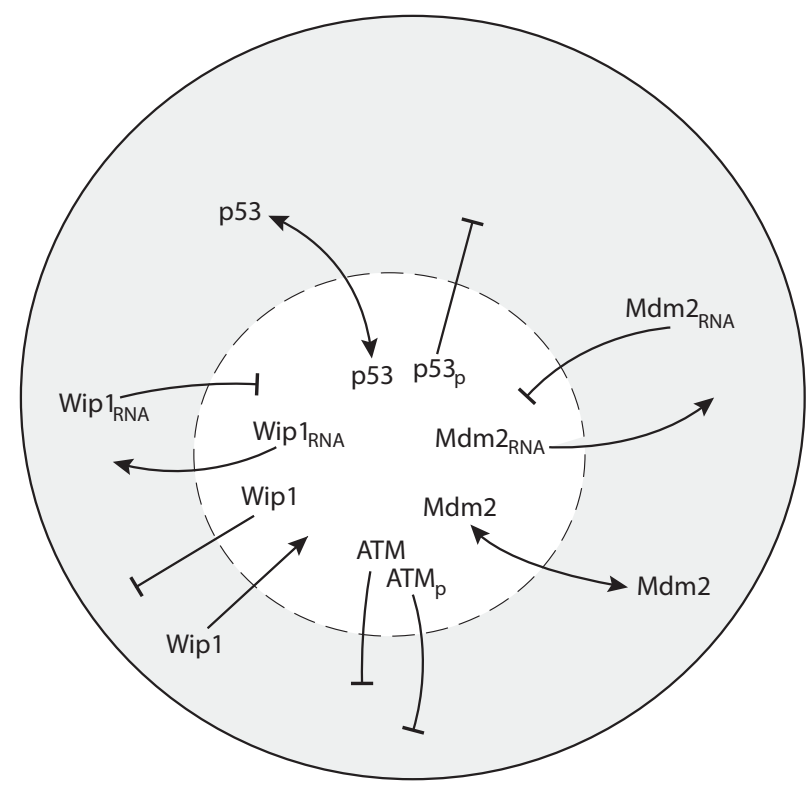

Figure 4.1: Assumptions on the location and exchange of considered proteins between the nucleus and the cytoplasm made in our model. mRNA variables are denoted on this figure by the additional subscript $R N A$, and similarly the subscript $p$ denotes a phosphorylated protein. The proteins p53 and Mdm2 are assumed to migrate freely (i.e., without diffusion limitations, resulting in homogeneous concentrations) within each of the two compartments, nucleus and cytoplasm.

the overall p53 degradation [37, 38]. Thus, in parallel to Mdm2 ubiquitination labelling of p53, we add a normal decay term for the degradation of the cytoplasmic p53.

The genes coding for Mdm2 and Wip1 are expressed in a p53-dependent manner, and we have chosen to model the transcription of these genes quite classically, by using a Hill function with coefficient 4 , since the transcriptionally active p53 appears in tetrameric form [39]. Note that other choices of the coefficient can be used; however, different Hill coefficients may result in different dynamical responses of the studied system. Denoting the basal Mdm2 mRNA production rate $k_{S m}$, the protein transcription of the Mdm2 gene to its mRNA, $M d m 2_{R N A}$, can be written as

$$
\frac{d\left[M d m 2_{R N A}\right]}{d t}=k_{S m}+\underbrace{k_{S_{p m}} \frac{\left[p 53_{p}\right]^{4}}{\left[p 53_{p}\right]^{4}+K_{S_{p m}}^{4}}}_{\text {Mdm2 gene transcription }} .
$$


The mRNA of Mdm2 then moves to the cytoplasm where it is translated. Translation is modelled as a linear process with the constant translation rate $k_{t m}$. Note, however, that the equations for the cytoplasmic concentrations of mRNAs, Equation (B.2) in the appendix, describe the concentrations of the unbound mRNAs present in the cytoplasm. In other words, the mRNA of Mdm2 (similarly, the mRNA of Wip1) already used in translation is modelled as a loss of the free mRNA available in the cytoplasm, and thus it is subtracted from the total mRNA. Produced protein Mdm2 can then freely migrate between the cytoplasm and the nucleus, and ubiquitinate $p 53$ in both compartments (the equation for p53 in the cytoplasm is designed in the same way as for the p53 nuclear equation). Regulation of Mdm2 is represented only by considering degradation terms for $M d m 2$ and $M d m 2_{R N A}$. The constants $k_{S_{p m}}$ and $K_{S_{p m}}$ are, respectively, p53-dependent Mdm2 mRNA transcription velocity and Michaelis-Menten p53-dependent Mdm2 mRNA transcription rate.

Transcription and translation of the Wip 1 gene run similarly with the corresponding reaction constants $k_{S_{p w}}$ and $K_{S_{p w}}$, the production rate $k_{S w}$, and the translation rate $k_{t w}$. Note, however, that Wip 1 has been observed to be a predominantly nuclear protein [23]; for this reason, unlike Mdm2, the Wip1 protein has been assumed to move only to, and not from, the nucleus. Phosphatases other than Wip1 that can dephosphorylate ATM and p53 have not been considered in the model.

The compartment-specific physiological roles of the proteins, already mentioned in Section 3.4, translate into mathematical simulations in the model that explicitly take into account cell compartmentalisation. Our assumptions about the location of these proteins are shown on Figure 4.1.

Most of the kinetic velocities and Michaelis-Menten constants (Table B.1 in Appendix) appearing in the reactions are taken from the sicentific literature on the subject [5]; ATM and p53 dephosphorylation rates by Wip1 are taken from works of Shreeram et al. $[15,16]$. Other unknown parameters (in Wip1 expression, ATM activation) are chosen by exploring the space of parameters so that the system exhibit oscillatory dynamics for the proteins. These biological observation facts, together with linear or Michaelis-Menten degradation terms, and boundary conditions on protein exchanges through the nucleocytoplasmic membrane (their fluxes being taken as proportional to the differences between averaged nuclear and cytoplasmic concentrations $[5,6])$, finally result in the ODE system that is solved numerically (equations B.1 and B.2 in Appendix). 


\subsubsection{Short overview of other existing models}

There are several mathematical models describing the p53/Mdm2 dynamics, for example the already mentioned models by Lev Bar-Or et al. [19] which show (experimentally and numerically) the presence of damped oscillations. The model developed by Batchelor et al. [14], which is actually based on former models by Geva-Zatorsky et al. [18], includes only nuclear concentrations of proteins. Ma et al. $[40,41]$ use delay differential equations (DDE) to simulate particular delays in representing the transcription of Mdm2 mRNA and translation of Mdm2 mRNA into the Mdm2 protein. Their models contain p53 activation by ATM kinase and only nuclear species are considered.

Ciliberto et al. [42] use the negative feedback $p 53 \rightarrow M d m 2 \dashv p 53$ supplemented by a simplified positive feedback to reconstruct p53/Mdm2 oscillations. In the positive feedback, p53 initiates activation of a cascade of protein interactions (involving PTEN, PIP2, PIP3 and Akt) leading to temporal inhibition of cytoplasmic Mdm2 translocation to the nucleus. T. Zhang et al. [43] explore the mechanism of p53/Mdm2 network of [42] and offer three other models simulating $\mathrm{p} 53 / \mathrm{Mdm} 2$ dynamics which combine different positive feedbacks with the p53/Mdm2 negative feedback. In addition, they propose a mechanism enabling a cell to decide between cell cycle arrest and apoptosis, assuming that persistent p53 pulses trigger apoptosis. X.P. Zhang et al. [44] combine results of [42, 43] and develop a two-phase switch model, which includes p53/Mdm2, ATM/p53/Wip1 and p53/PTEN/Akt/Mdm2 feedbacks, and which can simulate irreversible transition from cell cycle arrest to apoptosis. Both models of [43, 44] simulating cell fate decision involve transcription of proarrest and proapoptosis proteins p21 and p53DINP1 (and other proteins), respectively, the activation of which, however, is regulated by the p53 affinity for the protein genes, i.e., proarrest proteins are produced initially in an early cell response phase to DSBs, and proapoptotic proteins are produced later after a few p53 pulses. Note that the transcriptional regulation of proteins is modelled by using Hill functions, mostly with coefficients 3 and 4, in all the mentioned works [40, 41, 42, 43, 44].

There are also some other models simulating p53 signalling network, for example, a recently proposed deterministic model by J.K. Kim and T.L. Jackson [45] with the positive feedback between p53 and Ror $\alpha$ and a model proposed by K. Puszyński et al. [46] taking stochastic effects into account. Sturrock et al. [47, 48, 49] and Dimitrio et al. [5, 6] study p53/Mdm2 dynamics, without Wip1, but using PDE spatial models with exchanges through the nucleocytoplasmic membrane, ATM being considered in Dimitrio et al. as a bifurcation parame- 
(a)

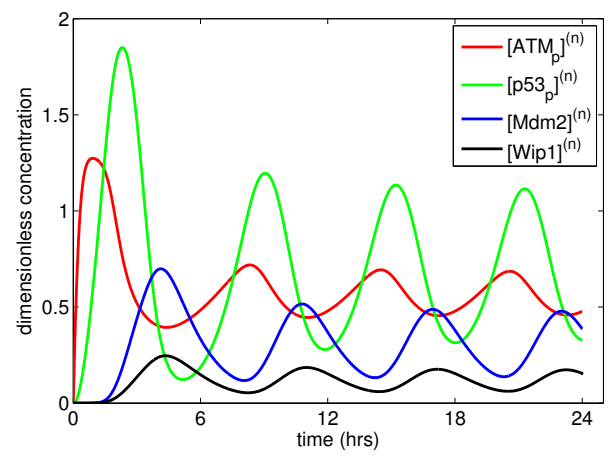

(b)

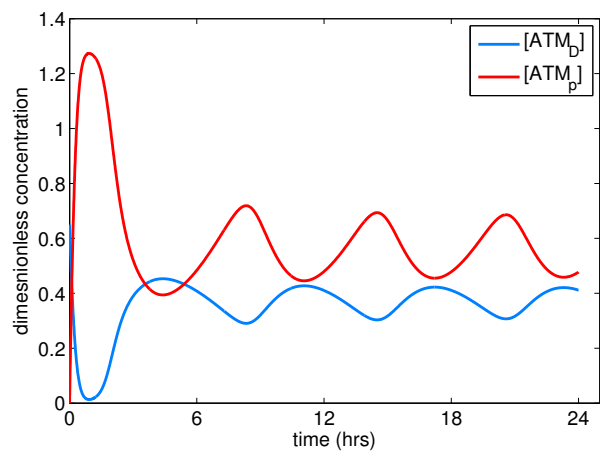

Figure 4.2: (a) Nuclear ATM/p53/Mdm2/Wip1 oscillations over a 24 hour-long time interval. (b) Evolution of the ATM monomerisation and dimerisation with the total concentration of ATM, $A T M_{T O T}(0)=1.3 \mu M$, and $[E]=0.1 \mu M$ (where $E$ is a hypothetical damage signalling molecule launching ATM activation). Plotted concentrations are dimensionless.

ter; both models exhibit sustained oscillations for p53.

\subsection{Numerical simulations and discussion}

\subsubsection{The ODE model reproduces the pulsatile behaviour of proteins}

Under specified circumstances, the model ODE system yields expected pulsatile behaviour for the proteins. Pulses of the involved proteins are illustrated on Figure 4.2. Figure 4.2(a) confirms that the model can be used to reconstruct experimental observations [14]. After DNA damage producing a (likely molecular) signal $E$ with concentration $[E]=0.1 \mu M$ (in this particular simulation), ATM is firstly activated. Then $A T M_{p}$ phosphorylates p53 and thus, the peak on the curve of $p 53_{p}$ evolution is observed later than the peak of $A T M_{p} . \operatorname{Mdm} 2$ and Wip1 are the targets of active $p 53_{p}$, hence the corresponding peaks appear after the $p 53$ peak. Mdm2 and Wip1 then regulate $\mathrm{p} 53$, which leads to $p 53_{p}$ dephosphorylation and degradation. Wip1 also dephosphorylates $A T M_{p}$ with formation of ATM dimers. The period of the first pulse for nuclear $p 53_{p}$ is $\sim 5.16$ hours and for $A T M_{p}$, it is $\sim 4.4$ hours, which fits experimental observations [14, 17]. ATM evolution can be seen on Figure 4.2(b). The amount of activated $A T M_{p}$ at the peaks of the evolution curve exceeds $50 \%$ of all ATM protein, as has been observed in biological experiments [24]. Note that the total concentration of ATM is set to $A T M_{T O T}=1.3 \mu M$ in this simulation. 
(a)

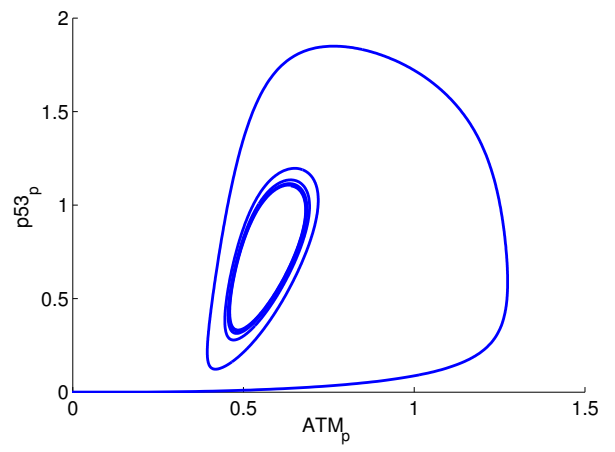

(b)

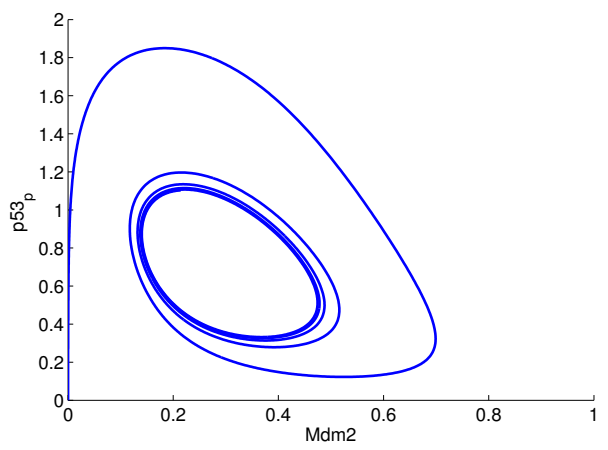

Figure 4.3: Phase plane curves relative to the involved nuclear proteins with $[E]=$ $0.1 \mu M$ (where $E$ is a hypothetical damage signalling molecule launching ATM activation): (a) $A T M_{p}$ and $p 53_{p}$ (b) $M d m 2$ and $p 53_{p}$.

The pulses of the proteins on Figure 4.2 are, apart from a couple of first pulses, of the same amplitude, i.e., the model quickly produces sustained (undamped) oscillations [14, 17]. This can be seen from the phase plane Figures 4.3 (and Figures E.1 in the Appendix), showing orbits of the solutions fastly converging toward stable limit cycles. The first pulses on Figure 4.2(a) are of higher amplitude mainly because of the choice of initial concentrations of proteins; all initial concentrations of proteins are set to be zero in our simulations, and since Wip1 and Mdm2 are not initially present in the cell, the concentrations of p53 and ATM increase until the regulators Wip1 and Mdm2 start to accumulate in the nucleus and thus inhibit further p53 and ATM production.

The proposed ODE model is "noise-free" and we do not consider stochasticity in protein gene expression, nor noise in protein production rates. The amplitudes of experimentally observed pulses in the extended research works of GevaZatorsky et al. [18] are found to vary widely from peak to peak and from cell to cell (even within the same irradiation dose), mainly due to noise in protein production rates. It is also worth mentioning that the periods of biologically measured oscillations are affected by noise, so that the duration of one pulse may be different from another; however, this difference comes with a variability of (at most) $20 \%$ in contrast to the $70 \%$ variability in amplitudes [18]. Our ODE model shows variability in periods in the first couple of pulses, simulated with $[E]=0.1 \mu M$ (Figure E.2(a) in the Appendix). The first pulse is of length 5.16 hours, the period of the stable limit cycle for $[E]=0.1 \mu M$ is $\sim 6$ hours. We leave the study of the 
impact of noise on oscillations for further modelling works.

\subsubsection{Bifurcation analysis of the system reveals two supercritical Hopf bifurca- tion points that correlate with DNA damage levels}

(a)

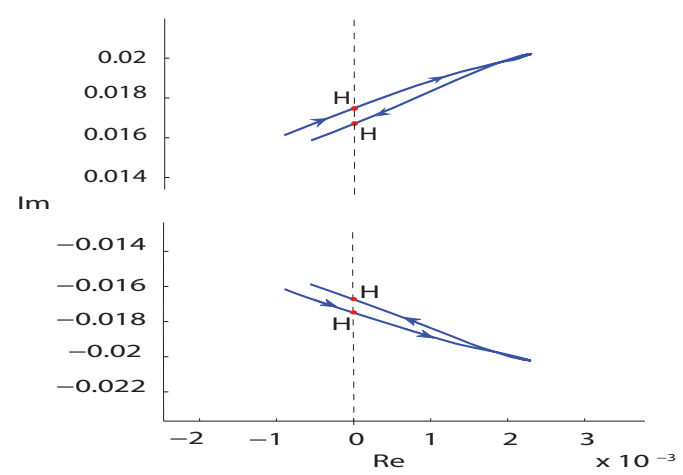

(b)

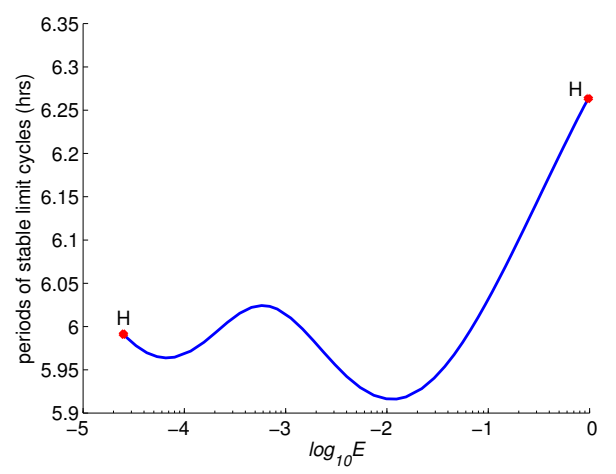

Figure 4.4: (a) Pair of eigenvalues crossing the imaginary axis for $[E]=2.5 \times 10^{-5}$ and $[E]=0.97$ and thus revealing Hopf bifurcation points. (b) Evolution of the period of the stable limit cycles occurring between the two Hopf bifurcation points; $[E]$ is in logarithmic scale.

With $[E]=0$ we can easily calculate the equilibrium point of the studied system (not shown). Forward continuation, using the Matlab package MatCont [50], of the equilibrium curve starting from the previously computed equilibrium point, numerically reveals two Hopf bifurcation points with negative Lyapunov coefficients [51] p. 120, i.e., corresponding to supercritical Hopf bifurcations. These Hopf bifurcation points are obtained for the values $[E]_{1}=2.5 \times 10^{-5} \mu M$ and $[E]_{2}=0.97 \mu M$. Note that the computed equilibria are hyperbolic, [51] p. 67, except for the two Hopf bifurcation points, since the Jacobian matrix of the system has eigenvalues with non-zero real parts for all values of $[E]$ except $[E]_{1}$ and $[E]_{2}$. A special situation appears in case of the Hopf points where a pair of complex eigenvalues crosses the imaginary axis, see Figure 4.4(a). For values below $[E]_{1}$ the equilibrium of the system is stable (all eigenvalues have negative real parts) and the concentrations of proteins tend to their steady states very quickly. By passing through this value, the equilibrium becomes unstable (the pair of complex eigenvalues crossing the imaginary axis change the real parts from negative to positive) and stable limit cycles appear; the equilibrium of the ODE system is stable again for $[E]>[E]_{2}$ (all eigenvalues have negative real parts again). 
The periods of the stable limit cycles vary between 5.92 and 6.26 hours with varying $[E]$, as illustrated on Figure $4.4(\mathrm{~b})$. Bifurcation diagrams of $p 53_{p}$ with respect to $E, p 53_{p} / A T M_{p}, p 53_{p} / M d m 2$ and $p 53_{p} / A T M_{p}$ are plotted on Figures 4.5. The amplitudes of $p 53_{p}$ and $A T M_{p}$ of the stable limit cycles are small for values of $[E]$ close to $[E]_{1}$ and $[E]_{2}$, respectively (as expected in the case of supercritical Hopf bifurcations), which is illustrated on Figures 4.5(a) and 4.5(b). This does not, however, mean that the amplitudes of concentrations are of such small values throughout the whole time period. In fact, it may take several days until the limit cycle is reached; compare, for example, Figures 4.5(a) with 4.6(a) and 4.6(b).

In our ODE settings, $E$ is considered as the measure of DNA damage. Interesting situations appear whenever $E$ is small and close to the first Hopf bifurcation point (which may correspond to a few DSBs only) and when $E$ is very big (which may correspond to serious DNA damage and many DSBs). In the first case, the $A T M_{p}$ solutions do not even oscillate for the values of $[E] \approx[E]_{1}$. The ATM trajectories then tend to steady states with low values; however, these small concentrations of $A T M_{p}$ can still elicit $p 53_{p}$ pulses, see Figure 4.6(a). This may contradict experimental observations that show ATM oscillations accompanying p53 oscillations after DNA damage [14]; note, however, that those experiments were performed with exposure of cells to $10 \mathrm{~Gy}$ of $\gamma$-irradiation and did not consider that DNA damage can cause only a few DSBs (and which may correspond to $\left.[E]_{1}\right)$. On the other hand, other works show evidence that only very small amounts of ATM (even not detectable) can still elicit actual p53 signalling [22, 26]. Indeed, DNA damage at $[E] \approx[E]_{1}$ may correspond to occasional DNA DSBs which do not need ATM for DNA repair and only moderate ATM is produced which is still efficient in p53 activation.

ATM oscillations are damped and disappear for $[E]$ greater than $[E]_{2}$ : once the oscillations have vanished, they do not appear again with further increasing values of $[E]$. Figure 4.6(b), for example, shows the protein dynamics for $[E]=10 \mu M$. In this case ATM is almost fully activated and only small deviations in ATM concentration can be seen. Hence, we can speculate that if it is impossible to repair DNA DSBs, then phosphatase activities on ATM are inhibited, active ATM achieves its maxima and apoptosis is initiated. However, the latter suggestion is not supported by experimental observations; in fact, there is no paper in the literature, to our best knowledge, clarifying ATM activity and location in single cells committing apoptosis. Thus, ATM oscillates whenever it is necessary for a proper cell response to DNA damage of an alive cell, and once $[E]$ becomes too high, oscillations disappear and the cell is sent to apoptosis and dies. Such interpretation, however, requires $E$ to be dependent not only on DNA damage levels but also 
(a)

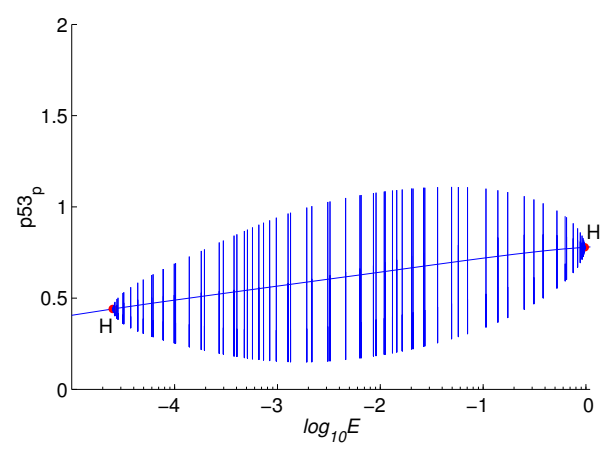

(c)

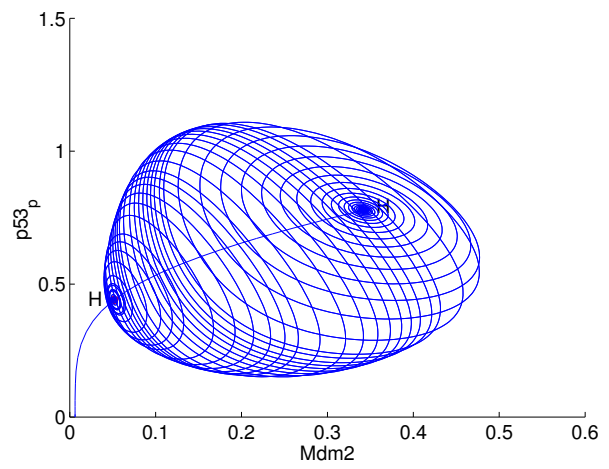

(b)

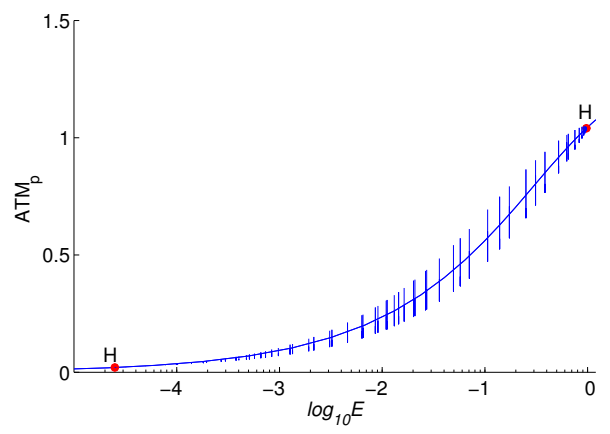

(d)

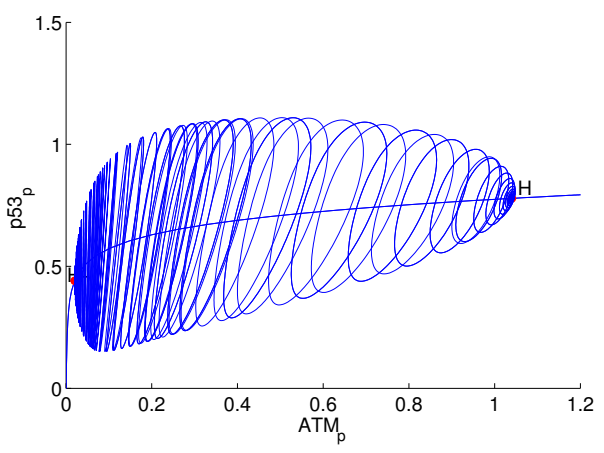

Figure 4.5: (a) Bifurcation diagram for nuclear $p 53_{p}$, (b) bifurcation diagram for nuclear $A T M_{p}$ with respect to $E=[E], E$ is in logarithmic scale. Bars plotted on figures are the heights (showing maximum and minimum) of the amplitudes of stable limit cycles. (c) $p 53_{p} / M d m 2$ limit cycles occurring for values of the damage signal $E$ between the two Hopf bifurcation points. (d) The same in the $p 53_{p} / A T M_{p}$ phase plane. Plotted concentrations are dimensionless. The equilibrium curves in (c) and (d) are the curves joining the two Hopf bifurcation points $\mathrm{H}$, constituting stable, then unstable, and then stable equilibrium branch again, with the stable limit cycles surrounding the unstable equilibrium points. 
(a)

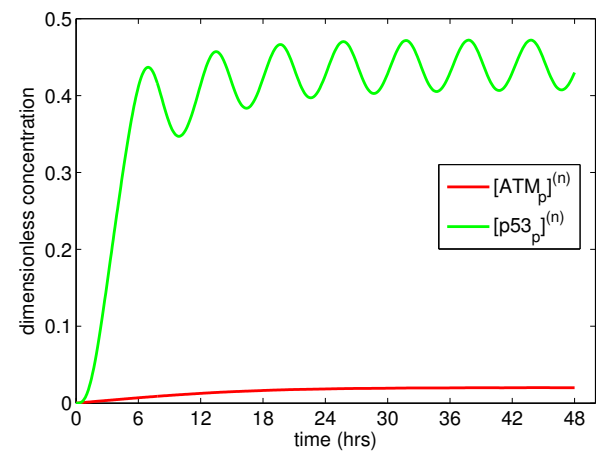

(b)

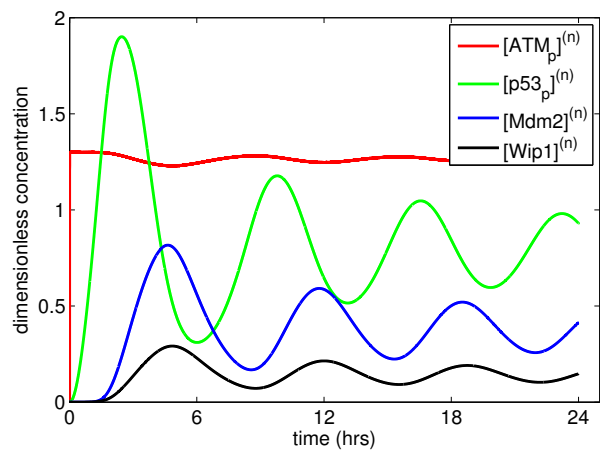

Figure 4.6: (a) Nuclear $p 53_{p} / A T M_{p}$ concentrations from the ODE system for $[E]=[E]_{1}=2.5 \times 10^{-5} \mu M$. The concentration of $A T M_{p}$ converges to the equilibrium point and the concentration of $p 53_{p}$ tends to a stable limit cycle. (b) Nuclear $A T M_{p} / p 53_{p} / M d m 2 / W i p 1$ concentrations for $[E]=10 \mu M$. The concentration of $A T M_{p}$ is of small amplitude, getting even smaller with further increasing $[E]$. Plotted concentrations are dimensionless, $A T M_{T O T}(0)=1.3 \mu M$.

on other factors amplifying the abundance of $E$, possibly with the contribution of proapoptotic proteins. Indeed, Figure 4.6(b) still shows (damped) p53 pulses, initially of higher amplitudes, that can act transcriptionally on its substrates.

\subsubsection{Two negative feedback loops and a compartmental distribution of cellular processes produce sustained oscillations}

In order to prove that the oscillating ATM protein plays an active role in achieving p53 pulses we made several simulations to test different possibilities and thus we explored the system in more details. For example, the model can be used to mimic the observed inhibition of $\mathrm{p} 53 / \mathrm{Mdm} 2$ pulses following one full ATM pulse [14]; this is illustrated on Figures 4.7, where ATM is inhibited after one pulse. We can see the complete first pulses of ATM, p53, Mdm2 and Wip1, among which protein Wip1 blocks further ATM activation (in the corresponding equation (4) of ATM dephosphorylation by Wip1, the Michaelis-Menten constant $K_{d p h 2}$ is set to be very small, $\left.K_{d p h 2}=0.0001\right)$. Although the first pulses of the p53 and Mdm2 proteins are induced, they are not sufficient to produce subsequent pulses after ATM dissociation is blocked.

Other tests when we inhibit, respectively,

- the negative feedback Wip1 $\dashv A T M\left(k_{d p h 2}=0\right.$ in (4), Figure E.3(a) in 
(a)

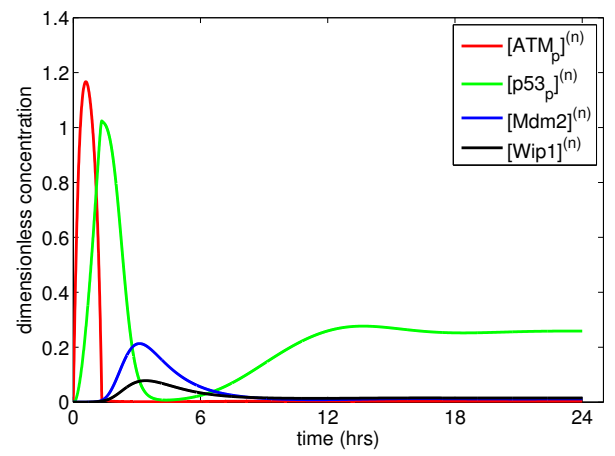

(b)

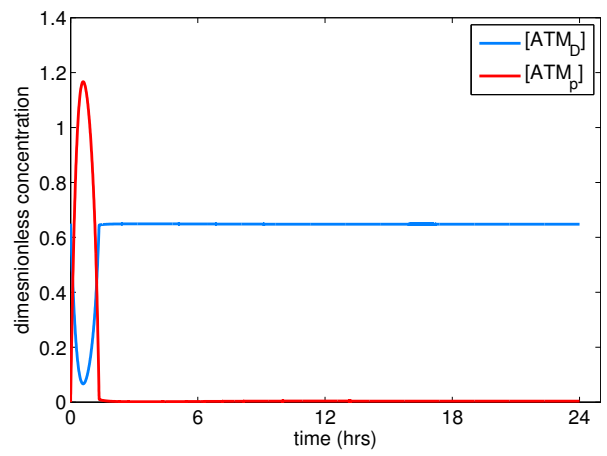

Figure 4.7: (a) The nuclear ATM/p53/Mdm2/Wip1 dynamics over a 24 hour-long time interval following ATM inhibition after one full pulse. (b) Evolution of the ATM monomerisation and dimerisation with the total concentration of ATM, $A T M_{T O T}(0)=1.3 \mu M$, and $[E]=0.1 \mu M$, following ATM inhibition after one full pulse. Plotted concentrations are dimensionless.

Appendix),

- the positive feedback $A T M \rightarrow p 53\left(k_{3}=0\right.$ in (6), Figure E.3(b)),

- the negative feedback Wip $1 \dashv p 53_{p}\left(k_{d p h 1}=0\right.$ in (6), Figure E.3(c)),

- the positive feedback $p 53_{p} \rightarrow$ Wip1 $\left(k_{S_{p w}}=0\right.$, Figures E.3(d) and (e)),

produce either damped oscillations or do not produce oscillations at all. The ATM and Wip1 proteins must thus be involved in the $\mathrm{p} 53 / \mathrm{Mdm} 2$ dynamics in order to produce sustained p53 oscillations.

The p53/Mdm2 negative feedback cannot be omitted either, since the inhibition of Mdm2 activity on p53 ( $k_{1}=0$ in (6), see Figure 4.8(a)) or the inhibition of the transcriptional activity of $p 53_{p}$ on $\operatorname{Mdm} 2\left(k_{S_{p m}}=0\right.$ in (7), Figures E.3(f)), does not produce oscillations.

We can further ask whether the two feedback loops without strict location of proteins in the compartments can lead to p53 oscillations. Merging protein dynamics into one compartment (the whole cell), i.e. omitting exchange of proteins between the nucleus and the cytoplasm but rather considering both compartments as the one where proteins and their mRNA can freely migrate, is not, however, sufficient in producing sustained oscillations. Figure 4.8(b) shows evolution of 
protein concentrations in the case $[E]=0.1 \mu M$. As in the case shown on this figure, all other choices of $[E]$ exhibit concentrations of proteins converging to their steady states (bifurcation analysis does not reveal any significant point). The role of compartmentalisation in $\mathrm{p} 53$ dynamics has been studied in more details in another research work [5, 52].

Hence, the feedbacks $p 53 \rightarrow$ Mdm $2 \dashv p 53$ and ATM $\rightarrow$ p53 $\rightarrow$ Wip $1 \dashv$ ATM together with compartmental distribution of proteins result in sustained oscillations, and neglecting any part of these three components fails to produce sustained oscillations.

(a)

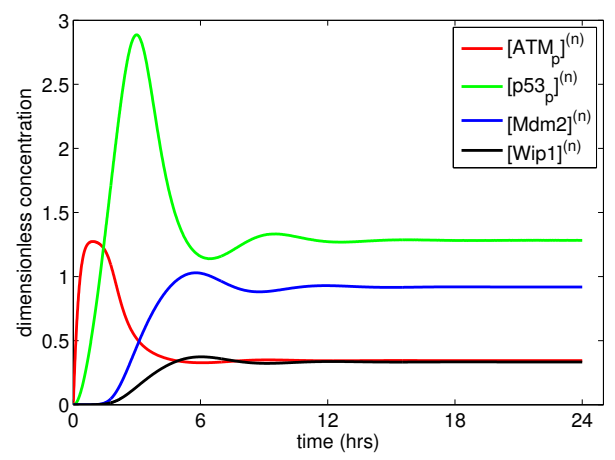

(b)

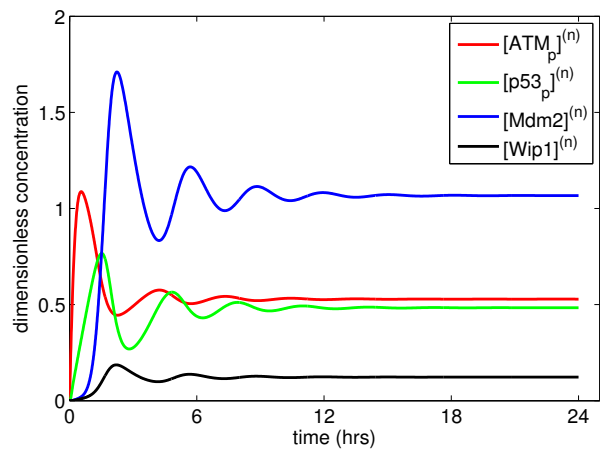

Figure 4.8: (a) The ATM/p53/Mdm2/Wip1 dynamics over a 24 hour-long time interval, $[E]=0.1 \mu M$, in a case when Mdm2-mediated ubiquitination of p53 is inhibited, $k_{1}=0$. (b) The ATM/p53/Mdm2/Wip1 dynamics over a 24 hourlong time interval and $[E]=0.1 \mu \mathrm{M}$ in a case when only one compartment is considered.

\subsubsection{ATM threshold required for initiation of $p 53$ pulses}

Note again that there are two significant points in the range of the signal $E$, the two Hopf bifurcation points, $[E]_{1}=2.5 \times 10^{-5} \mu M$ and $[E]_{2}=0.97 \mu M$ in the presented simulation settings: values of $E$ starting from $[E]_{1}$ are sufficient to elicit sustained oscillations in the p53 protein nuclear concentrations, and the second Hopf bifurcation point $[E]_{2}$ is the critical point at which stable limit cycles (otherwise said, sustained oscillations of the proteins) disappear. This point thus may mark a decision for the cell not to go on in DNA DSBs repairing processes, and rather to start apoptosis. A relation to a supposed apoptotic threshold [1] remains however to be further established. 
The hypothetical signal $E$ is supposed to be produced by DNA DSB sensors and/or by changes in chromatin structure, and may be affected by other factors like the MRN complex bound to the DNA break sites and the presence of proarrest and proapoptotic proteins. Then $E$ is delivered to ATM dimers and promotes ATM activation. Interestingly, an amount of $E$ as small as $[E]_{1}$ can, for a value of the total ATM protein $A T M_{T O T}=1.3 \mu M$, activate ATM at the concentration $\sim 0.02 \mu M$, Figure 4.6(b), that is sufficient to produce oscillations in p53.

Note, however, that the Hopf bifurcation points are dependent on the total concentration of nuclear ATM, AT $M_{T O T}$. Indeed, Figure 4.9(a) shows the evolution of the Hopf bifurcation points with respect to $A T M_{T O T}$ (on the y-axis). We can see that the values of $[E]_{1}$ do not change dramatically and they range between $1,17 \times 10^{-5}$ (which corresponds to $A T M_{T O T}=10 \mu M$ ) and $3.5 \times 10^{-5}$ (which corresponds to $A T M_{T O T}=0.78 \mu M$ ). The dependence of $[E]_{2}$ on $A T M_{T O T}$ seems to be more intriguing. Similarly to $[E]_{1}$ the Hopf bifurcation points $[E]_{2}$ increase with decreasing $A T M_{T O T}$, and asymptotically reach values corresponding to $A T M_{T O T} \approx 0.77 \mu M$, Figure 4.9(a). In other words, the system has two Hopf bifurcation points for $A T M_{T O T}>0.77 \mu M$ (with similar bifurcation diagrams between these points Figure 4.9(b)); the only one Hopf bifurcation point $\sim 3.8 \times 10^{5}$ below $A T M_{T O T}=0.77 \mu M$ determining stable solution and unstable solution (stable limit cycle). The system does not have any Hopf bifurcation point for $A T M_{T O T}<0.082 \mu M$.

Interestingly, for all the $[E]_{1}$ values corresponding to different $A T M_{T O T}$, the concentration in activated ATM protein always reaches the steady-state value $0.02 \mu M$ (not shown). This helps resolve a biological unanswered question; in particular, it has been observed that only a fraction of ATM molecules can be sufficient to initiate proper cell responses to DNA damage [22]; however, a certain threshold for initial ATM concentration is supposed to exist (but it is not specified) to initiate the ATM signalling pathway [14]. Thus, the analysis of our model reveals that the threshold for ATM, which is independent of the total nuclear ATM proteins, can be $0.02 \mu M$ (in our model constant settings).

\section{Conclusion}

Explaining experimentally observed p53 oscillations in human cancer cells after exposure of the cells to $\gamma$-irradiation $[14,17,18]$ is a mathematical modelling challenge, especially in the perspective of proposing p53-mediated anticancer drug-induced cell cycle arrest/apoptotic predictions. This paper is the extension of a previous work by Dimitrio et al. $[5,6]$. It presents the dynamics of the 
(a)

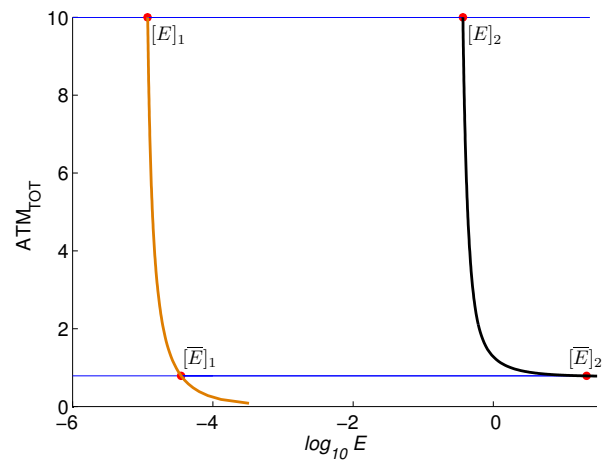

(b)

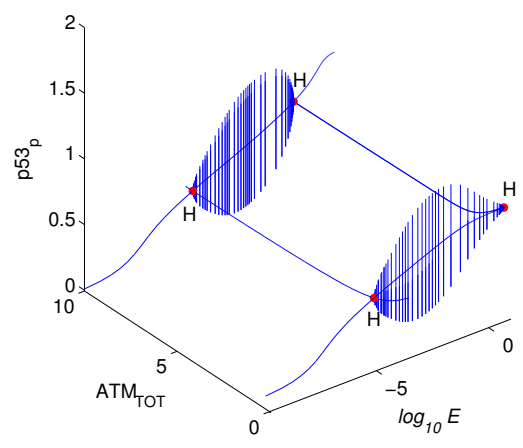

Figure 4.9: (a) The evolution curves of both Hopf bifurcation points (curves in brown and black colours). The Hopf bifurcation points $[E]_{1}$ and $[E]_{2}$ are computed for $A T M_{T O T}=10 \mu M$, the points $[\bar{E}]_{1}$ and $[\bar{E}]_{2}$ for $A T M_{T O T}=0.78 \mu M$. The concentration $E=[E]$ is in the logarithmic scale. (b) Bifurcation diagrams of $p 53_{p}$ with respect to changing $A T M_{T O T}$ and $[E]$.

"guardian of the genome" p53, that plays essential parts in cell responses to DNA damage and checkpoint signalling. The spatial distribution of proteins in a cell is taken into account and it is shown that such compartmental recognition of cellular events allows to reconstruct the pulsatile behaviour of proteins without the need to introduce any time delay in their dynamics.

The oscillatory dynamics of the involved proteins can be speculatively explained as periodical testing of the presence of DNA damage; if unrepaired DNA DSBs still exist, additional pulses of both phosphorylated ATM and p53 are triggered. More precisely, we propose the following interpretation: the level of phosphorylated ATM increases in response to DSBs and is then reduced due to the increase of the p53 concentration (since ATM phosphorylation of p53 leads to p53 stabilisation, which, afterwards, activates Wip1, that inactivates ATM). When p53 levels are in turn reduced by the interaction with Mdm2 (after p53 dephosphorylation by Wip1), ATM is released to re-examine the DNA. If the number of breaks remains above a certain threshold, the pathway becomes reactivated, leading to a second pulse of ATM and p53, and this continues until the number of DNA breaks is dropped below the threshold, or the cell decides to start apoptosis [14].

The original models of Dimitrio et al. [5, 6] involve ATM as a direct measure of DNA damage, in the form of a parameter that remains constant in the 
studied time interval. It has been experimentally shown, however, that a pulsatile behaviour for p53/Mdm2 cannot be achieved without oscillations of ATM [14]. In addition, there exist biological studies that confirm a role for ATM in cancer cell apoptosis induced, for example, by ionising radiations [53]. Hence, in this extended modelling work, we consider ATM as an essential part of the DNA damage signalling pathway, varying with time, that after ATM dimer dissociation and activation, phosphorylates p53 and thus initiates p53 transcriptional activity towards proarrest and proapoptotic genes (for example, p21 and 14-3-3 $\sigma$ on the proarrest side, and Pig3, Apaf1 and PUMA on the proapoptotic side).

The p53 protein responds to a variety of cellular stresses (such as those causing DNA strand breaks, e.g., cytotoxic drug insults) through the transcription mode of protein production. Despite the complexity of all signalling pathways occurring in the stressed cells and the production of proteins involved therein, we can conclude that the pathway including p53 activation can be successfully reconstructed by our compartmental model that, in particular, takes into account the ATM/p53/Mdm2/Wip1 pulsatile dynamics as has been observed in the scientific literature on the subject $[14,15,24,26]$, and as is summarised in details in the biological background part of the present work.

In an even more realistic vision of the processes leading a single cell to decision between survival and death, more than on deterministic events, random molecule encounters and stochastic gene expression should be taken into consideration, and should be modelled by stochastic processes (as sketched in [49] for Hes1), from which deterministic equations (partial differential equations physiologically structured in variables, identified as biological "readouts" in a single cell model, representing relevant biological variability inside the cell population) should be further designed at the level of cell populations.

In the same way, in the perspective of connecting the proposed p53 modelling with existing PK-PD models, drug effects, measured only at the cell population level, can be represented as environmental factors exerting their influence on molecular targets, included as functions in stochastic processes, at the level of a single cell. Establishing such connections between stochastic events at the individual cell level and resulting deterministic effects at the cell population level is a challenge for mathematical modelling that we intend to tackle in future works. Readers interested in such interconnections between stochastic and deterministic mathematics in the Hes 1 regulatory network are recommended to read the papers by $\mathrm{M}$. Sturrock et al. $[49,56]$ to get acquainted with this challenge, a considerable one in the most general case. 


\section{Appendix A. Equations for ATM dynamics}

Following in vivo observations [24], ATM is activated very shortly after DNA damage in the nucleoplasm, i.e., with no need of ATM binding DNA damage sites, through the dissociation of the inactive ATM dimers, $A T M_{D}$, into the active monomers, $A T M_{p}$. The activation signal is however not biologically specified; it is assumed to be produced by changes in chromatin after DNA damage [24, 27], amplified by MRN complex (sensor of DNA DSBs) [54], or this signal may be actually the MRN complex itself and its interaction with ATM [55]. Although there is not a general agreement among biologists, ATM is mostly thought to be activated very promptly. We thus represent ATM activation through its interaction with an unknown signal, likely a molecule or the result of a chain of molecular reactions, denoted by $E$, which is assumed to be constant in a very short time interval, so that the reaction of $E$ with ATM dimers can be written as the enzymatic reaction, Equation (2), i.e.

$$
E+A T M_{D} \underset{k_{-i}}{\stackrel{k_{i}}{\rightleftharpoons}} \text { Complex } \stackrel{k_{p h 2}}{\longrightarrow} 2 A T M_{p}+E .
$$

Dephosphorylation (and deactivation) of ATM monomers is observed through the interaction of $A T M_{p}$ with Wip1 $[14,15,16]$, Equation (3), i.e.

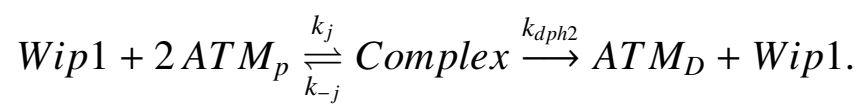

By applying the law of mass action and the quasi-steady-state approximation, the loss of $A T M_{D}$ derived from the first reaction (A.1) can be written in the form

$$
\frac{d\left[A T M_{D}\right]}{d t}=-k_{p h 2}[E] \frac{\left[A T M_{D}\right]}{K_{p h 2}+\left[A T M_{D}\right]} .
$$

However, the equation for the loss of $A T M_{p}$ is not so straightforward, since as a substrate we now have two $A T M_{p}$ proteins. Let us write

$$
s=\left[A T M_{p}\right], e=[\text { Wip } 1], p=\left[A T M_{D}\right] \text { and } c=[\text { Complex }]
$$

for the concentrations of the reactants in (A.2). Then the law of mass action applied to (A.2) leads to the following equation for the substrate $s$,

$$
\frac{d s}{d t}=-2 k_{j} s^{2} e+2 k_{-j} c
$$


since in every $c$ that is made, two of $s$ are used, and every time $c$ is degraded, two of $s$ are produced; and for the other reactants,

$$
\begin{aligned}
& \frac{d e}{d t}=-k_{j} s^{2} e+\left(k_{-j}+k_{d p h 2}\right) c, \\
& \frac{d c}{d t}=k_{j} s^{2} e-\left(k_{-j}+k_{d p h 2}\right) c, \\
& \frac{d p}{d t}=k_{d p h 2} c .
\end{aligned}
$$

The initial conditions are $e(0)=e_{0}, s(0)=s_{0}$ and $c(0)=p(0)=0$. With the conservation property of the enzyme $e, \frac{d e}{d t}+\frac{d c}{d t}=0$, and so with $e(t)=e_{0}-c(t)$, we have the system of ordinary differential equations (ODEs) reduced to

$$
\begin{aligned}
& \frac{d s}{d t}=-2 k_{j} s^{2}\left(e_{0}-c\right)+2 k_{-j} c, \\
& \frac{d c}{d t}=k_{j} s^{2}\left(e_{0}-c\right)-\left(k_{-j}+k_{d p h 2}\right) c .
\end{aligned}
$$

The quasi-steady-state approximation then assumes $\frac{d c}{d t} \approx 0$. Hence, from the second equation in (A.6) we can explicitly write $c$ in terms of $s$, in particular,

$$
c=\frac{e_{0} s^{2}}{s^{2}+K_{d p h 2}}
$$

where $K_{d p h 2}=\frac{k_{-j}+k_{d p h 2}}{k_{j}}$ is the Michalis-Menten rate of reaction. Substituting $c$ in the equation for $s$, we finally can write

$$
\frac{d s}{d t}=-2 e_{0} k_{d p h 2} \frac{s^{2}}{s^{2}+K_{d p h 2}},
$$

where in numerical simulations $e_{0}$ is replaced by $e$.

By coming back to our original problem reaction (A.2), we get the equation for the loss of $A T M_{p}$,

$$
\frac{d\left[A T M_{p}\right]}{d t}=-2 k_{d p h 2}[\text { Wip } 1] \frac{\left[A T M_{p}\right]^{2}}{\left[A T M_{p}\right]^{2}+K_{d p h 2}} .
$$

Finally, the combination of (A.3) and (A.9) can fully describe ATM activation 
and deactivation. In particular, equations of interest are

$$
\begin{aligned}
& \frac{d\left[A T M_{D}\right]}{d t}=-k_{p h 2}[E] \frac{\left[A T M_{D}\right]}{K_{p h 2}+\left[A T M_{D}\right]}+k_{d p h 2}[\text { Wip } 1] \frac{\left[A T M_{p}\right]^{2}}{K_{d p h 2}+\left[A T M_{p}\right]^{2}} \\
& \frac{d\left[A T M_{p}\right]}{d t}=2 k_{p h 2}[E] \frac{\left[A T M_{D}\right]}{K_{p h 2}+\left[A T M_{D}\right]}-2 k_{d p h 2}[\text { Wip } 1] \frac{\left[A T M_{p}\right]^{2}}{K_{d p h 2}+\left[A T M_{p}\right]^{2}}
\end{aligned}
$$

since the production of $A T M_{D}$ is half of $A T M_{p}$. Note that we do not consider ATM in inactive monomeric state.

Note that it is, however, not necessary to use both equations in numerical simulations, and actually, we cannot use both of them, since the Jacobian matrix of the ODE system composed of all other equations describing the p53/Mdm2 dynamics, see Appendix $\mathrm{B}$, is singular for $[E]=0$, i.e. for the value for which the system achieves equilibrium. This is because the derivative of the right-hand side of the first equation in (A.10) with respect to the variable $\left[A T M_{D}\right]$ is zero for $[E]=0$, similarly the derivatives of all other right-hand sides of the equations describing the dynamics of proteins are zeros as well (simply because these equations do not contain $\left[A T M_{D}\right]$, i.e. they are constants with respect to $\left[A T M_{D}\right]$ ); see the equations in (B.1) and (B.2). To calculate the equilibrium for $[E]=0$ is a straightforward algebraic exercise; the problem, however, is in the continuation of the equilibrium curve starting at the computed equilibrium for $[E]=0$, since continuation techniques require non-singular Jacobian matrices.

By recalling the conservation property of the ATM protein, i.e. $\left[A T M_{p}\right]+$ $2\left[A T M_{D}\right]=$ const $=A T M_{T O T}[\mu M]$ and thus by writing $\left[A T M_{D}\right]=\frac{1}{2}\left(A T M_{T O T}-\right.$ $\left.\left[A T M_{p}\right]\right)$ in the second equation in (A.10), we actually have the mathematical equation for $\left[A T M_{p}\right]$ in the form

$$
\begin{aligned}
\frac{d\left[A T M_{p}\right]}{d t}= & 2 k_{p h 2}[E] \frac{\frac{1}{2}\left(A T M_{T O T}-\left[A T M_{p}\right]\right)}{K_{p h 2}+\frac{1}{2}\left(A T M_{T O T}-\left[A T M_{p}\right]\right)} \\
& -2 k_{d p h 2}[\text { Wip } 1] \frac{\left[A T M_{p}\right]^{2}}{K_{d p h 2}+\left[A T M_{p}\right]^{2}} .
\end{aligned}
$$

The conservation property observed by Bakkenist and Kastan [24] claims (almost) non-changing concentration of the total ATM proteins. Note that ATM is not observed to be degraded in the cell and since it is the $370 \mathrm{kDa}$ large protein, its inactivation through the dimerisation instead of degradation is energetically more profitable. 


\section{Appendix B. The ODE system}

Section 4.1 contains the description of all processes and reactions which we have considered in the model. All the mentioned facts can be collected in the following equations, divided into those describing events occurring, respectively, in the nucleus and in the cytoplasm, Equations (B.1) and (B.2).

Protein exchange fluxes are modelled as linear contributions of the differences between averaged nuclear and cytoplasmic concentrations precisely determined by the permeability rates, see, for example, the term $p_{p} V_{r}\left([p 53]^{(n)}-[p 53]^{(c)}\right)$ in the first equation of (B.1). Here, $V_{r}$ is a special volume ratio introduced in [42] to underline the fact that reactions are $V_{r}$-times faster in the nucleus than in the cytoplasm provided that the nucleus is $V_{r}$-times smaller than the cytoplasm, and $p_{p}$ is the permeability coefficient for $\mathrm{p} 53[5,6]$.

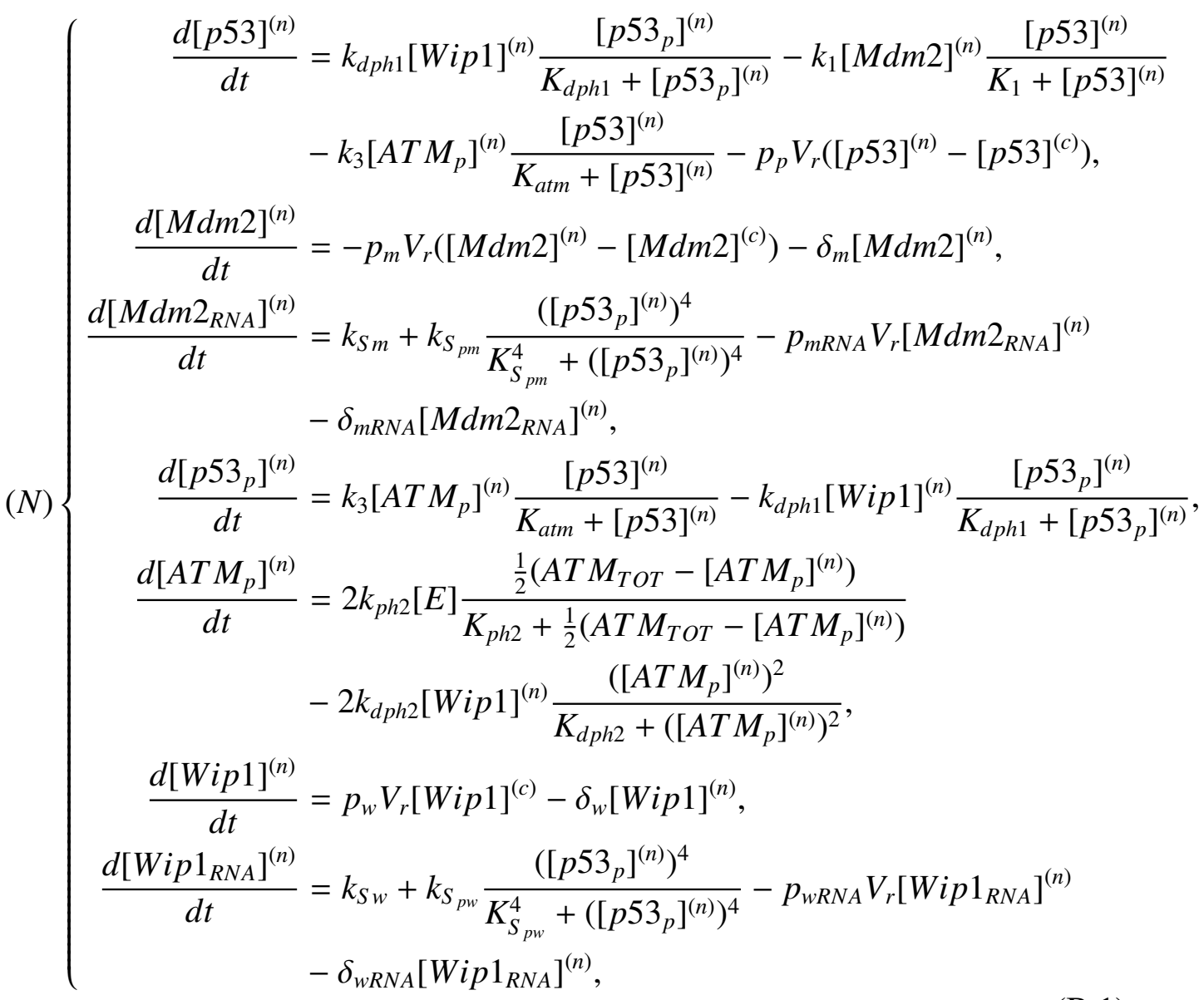


$(C)\left\{\begin{aligned} \frac{d[p 53]^{(c)}}{d t} & =k_{S}-k_{1}[M d m 2]^{(c)} \frac{[p 53]^{(c)}}{K_{1}+[p 53]^{(c)}}-p_{p}\left([p 53]^{(c)}-[p 53]^{(n)}\right)-\delta_{p}[p 53]^{(c)}, \\ \frac{d[M d m 2]^{(c)}}{d t} & =k_{t m}\left[M d m 2_{R N A}\right]^{(c)}-p_{m}\left([M d m 2]^{(c)}-[M d m 2]^{(n)}\right)-\delta_{m}[M d m 2]^{(c)}, \\ \frac{d\left[M d m 2_{R N A}\right]^{(c)}}{d t} & =p_{m R N A}\left[M d m 2_{R N A}\right]^{(n)}-k_{t m}\left[M d m 2_{R N A}\right]^{(c)}-\delta_{m R N A}\left[M d m 2_{R N A}\right]^{(c)}, \\ \frac{d[W i p 1]^{(c)}}{d t} & =k_{t w}\left[W i p 1_{R N A}\right]^{(c)}-p_{w}[W i p 1]^{(c)}-\delta_{w}[W i p 1]^{(c)}, \\ \frac{d\left[W i p 1_{R N A}\right]^{(c)}}{d t} & =p_{w R N A}\left[W i p 1_{R N A}\right]^{(n)}-k_{t w}\left[W i p 1_{R N A}\right]^{(c)}-\delta_{w R N A}\left[W i p 1_{R N A}\right]^{(c)},\end{aligned}\right.$

All numerical results presented in the text are, if it is not specified otherwise, produced by simulations with the constants listed in Table B.1.

\section{Appendix C. Equations for a one-compartment model}

In Section 4.2.3 we show that the two negative feedbacks together with the compartmental distribution of cell events are necessary to elicit protein pulses. In particular, Figure 4.8(b) confirms the need for two compartmental model. The system of equations which is tested in this one-compartmental model is in (C.1). 


\begin{tabular}{|c|c|c|c|}
\hline Parameter & Chosen Value & Units & Description \\
\hline$k_{d p h 1}$ & 78 & $\min ^{-1}$ & Wip1-dependent p53 dephosph. velocity \\
\hline$K_{d p h 1}$ & 25 & $\mu M$ & Mich.-Men. rate of Wip1-dependent p53 dephosph. \\
\hline$k_{3}$ & 3 & $\min ^{-1}$ & p53 phosphorylation velocity \\
\hline$K_{a t m}$ & 0.1 & $\mu M$ & Mich.-Men. rate of p53 phosph. \\
\hline$k_{1}$ & 10 & $\min ^{-1}$ & p53 ubiquitination velocity \\
\hline$K_{1}$ & 1.01 & $\mu M$ & Mich.-Men. rate of p53 ubiq. \\
\hline$p_{p}$ & 0.083 & $\min ^{-1}$ & p53 permeability \\
\hline$V_{r}$ & 10 & adim & Volume ratio \\
\hline$p_{m}$ & 0.04 & $\min ^{-1}$ & Mdm2 permeability \\
\hline$\delta_{m}$ & 0.16 & $\min ^{-1}$ & Mdm2 degradation rate \\
\hline$k_{S m}$ & 0.005 & $\mu M / \min$ & Basal Mdm2 RNA transcription rate \\
\hline$k_{S_{p m}}$ & 1 & $\mu M / \min$ & Mdm2 RNA transcription velocity \\
\hline$K_{S p m}$ & 0.1 & $\mu M$ & Mich.-Men. rate of Mdm2 RNA trans. \\
\hline$p_{m R N A}$ & 0.083 & $\min ^{-1}$ & Mdm2 RNA permeability \\
\hline$\delta_{m R N A}$ & 0.0001 & $\min ^{-1}$ & Mdm2 RNA degradation rate \\
\hline$k_{t m}$ & 1 & $\min ^{-1}$ & Mdm2 translation rate \\
\hline$k_{S}$ & 0.015 & $\mu M / \min$ & Basal p53 synthesis rate \\
\hline$\delta_{p 53}$ & 0.2 & $\min ^{-1}$ & p53 degradation rate \\
\hline$p_{w}$ & 0.083 & $\min ^{-1}$ & Wip1 permeability \\
\hline$\delta_{w}$ & 0.2 & $\min ^{-1}$ & Wip1 degradation rate \\
\hline$k_{S w}$ & 0.03 & $\mu M / \min$ & Basal Wip1 RNA transcription rate \\
\hline$k_{S_{p w}}$ & 1 & $\mu M / \min$ & Wip1 RNA transcription velocity \\
\hline$K_{S_{p w}}$ & 0.1 & $\mu M$ & Mich.-Men. rate of Wip1 RNA trans. \\
\hline$p_{w R N A}$ & 0.083 & $\min ^{-1}$ & Wip1 RNA permeability \\
\hline$\delta_{w R N A}$ & 0.001 & $\min ^{-1}$ & Wip1 RNA degradation rate \\
\hline$k_{t w}$ & 1 & $\min ^{-1}$ & Wip1 translation rate \\
\hline$k_{d p h 2}$ & 96 & $\min ^{-1}$ & Wip1-dependent ATM dephosph. velocity \\
\hline$K_{d p h 2}$ & 26 & $\mu M$ & Mich.-Men. rate of Wip1-dependent ATM dephosph. \\
\hline$k_{p h 2}$ & 15 & $\min ^{-1}$ & ATM phosphorylation velocity \\
\hline$K_{p h 2}$ & 1 & $\mu M$ & Mich.-Men. rate of ATM phosph. \\
\hline$[E]$ & 0.1 & $\mu M$ & Concentration of "the damage signal" \\
\hline$A T M_{T O T}$ & 1.3 & $\mu M$ & Total ATM concentration \\
\hline
\end{tabular}

Table B.1: Parameter values for the ODE model (B.1) and (B.2). 


$$
\left\{\begin{aligned}
\frac{d[p 53]}{d t} & =k_{S}+k_{d p h 1}[W i p 1] \frac{\left[p 53_{p}\right]}{K_{d p h 1}+\left[p 53_{p}\right]}-k_{1}[M d m 2] \frac{[p 53]}{K_{1}+[p 53]} \\
& -k_{3}\left[A T M_{p}\right] \frac{[p 53]}{K_{a t m}+[p 53]}-\delta_{p}[p 53] \\
\frac{d[M d m 2]}{d t} & =k_{t m}\left[M d m 2_{R N A}\right]-\delta_{m}[M d m 2] \\
\frac{d\left[M d m 2_{R N A}\right]}{d t} & =k_{S m}+k_{S_{p m}} \frac{\left(\left[p 53_{p}\right]\right)^{4}}{K_{S_{p m}^{4}}^{4}+\left(\left[p 53_{p}\right]\right)^{4}}-\delta_{m R N A}\left[M d m 2_{R N A}\right]-k_{t m}\left[M d m 2_{R N A}\right] \\
\frac{d\left[p 53_{p}\right]}{d t} & =k_{3}\left[A T M_{p}\right] \frac{[p 53]}{K_{a t m}+[p 53]}-k_{d p h 1}[W i p 1] \frac{\left[p 53_{p}\right]}{K_{d p h 1}+\left[p 53_{p}\right]} \\
\frac{d\left[A T M_{p}\right]}{d t} & =2 k_{p h 2}[E] \frac{\frac{1}{2}\left(A T M_{T O T}-\left[A T M_{p}\right]\right)}{K_{p h 2}+\frac{1}{2}\left(A T M_{T O T}-\left[A T M_{p}\right]\right)} \\
& -2 k_{d p h 2}[W i p 1] \frac{\left(\left[A T M_{p}\right]\right)^{2}}{K_{d p h 2}+\left(\left[A T M_{p}\right]\right)^{2}}, \\
\frac{d\left[W i p 1_{R N A}\right]}{d t} & =k_{S w}+k_{S_{p w}} \frac{\left(\left[p 53_{p}\right]\right)^{4}}{K_{S_{p w}}^{4}+\left(\left[p 53_{p}\right]\right)^{4}}-\delta_{w R N A}\left[W i p 1_{R N A}\right]-k_{t w}\left[W i p 1_{R N A}\right]
\end{aligned}\right.
$$

\section{Appendix D. Matlab code}

function ODEp53Mdm2ATMWip1system

$\%$... solves the ODE system (B.1) and (B.2) written in ODEs.m (below), $\%$ The ODE system involves ATM/p53/Mdm2/Wip1 protein dynamics.

$\%$

$\%$ This function returns the evolution of proteins' concentrations in the $\%$ time interval of 24 hours. Output are Figures 4.2(a) and (b).

$\%$ To get other plots of concentrations (Figures 4.6, 4.7, 4.8 and E.3), $\%$ please, change the constants as they are specified in the paper $\%$ (or as you wish if you are interested in other sorts of plots). $\%$ Note, however, that setting some parameters to zero may lead to $\%$ collisions with nondimensionalisation of variables (e.g. division by 
$\%$ zero and thus to incorrect results)!

$\%$

$\%$ Note, that the bifurcation analysis of the system is done through the $\%$ Matlab package MatCont.

timeint $=[0,1440] ; \%$ time interval

initcond $=\operatorname{zeros}(12,1) ; \%$ initial conditions

ATMtot $=1.3 ; \%$ total concentration of ATM proteins (monomers and dimers) $E=0.1 ; \%$ signal produced by DNA damage

$\%$ solve ODE

$[t, \mathrm{baru}]=\operatorname{ode} 45(\Theta(\mathrm{t}, \mathrm{u})$ ODEs $(\mathrm{t}, \mathrm{u}, \mathrm{ATMtot}, \mathrm{E})$, timeint, initcond $)$;

$\%$ plot solutions (Figures 4.2 )

figure;

axes ('FontSize', 14);

plot (t, (ATMtot-baru (: , 7))/2, 'Color', [ [0 0.5 1] , 'LineWidth', 2, . .

'DisplayName', ' [ATM_D]');

hold on;

plot ( $t, \operatorname{baru}(:, 7)$, 'Color', [1 00 0 , 'LineWidth', 2, . .

'DisplayName', ' [ATM_p] ');

hold off;

xlabel ('time (hrs)', 'FontSize', 14);

set (gca, 'XTickLabel', \{'0', '6', ' 12', '18' , '24'\}, 'XTick', [0 3607201080 1440]);

ylabel ('dimesnionless concentration', 'FontSize',14);

legend('show');

figure;

axes ('FontSize', 14);

plot (t, baru (: , 7), 'Color' , [1 00 0 , 'LineWidth', 2, . . .

'DisplayName', ' [ATM_p]^\{(n)\}');

hold on;

plot (t, baru (: , 4), 'Color', [0 10 l , 'LineWidth', 2, . .

'DisplayName', '[p53_p]^\{(n)\}');

plot (t, baru (: , 2), 'Color' , [0 001 , , 'LineWidth', 2, . . .

'DisplayName', ' [Mdm2] ^ $\left.\{(\mathrm{n})\}^{\prime}\right)$;

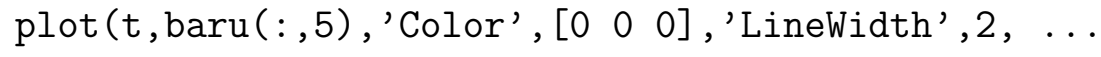




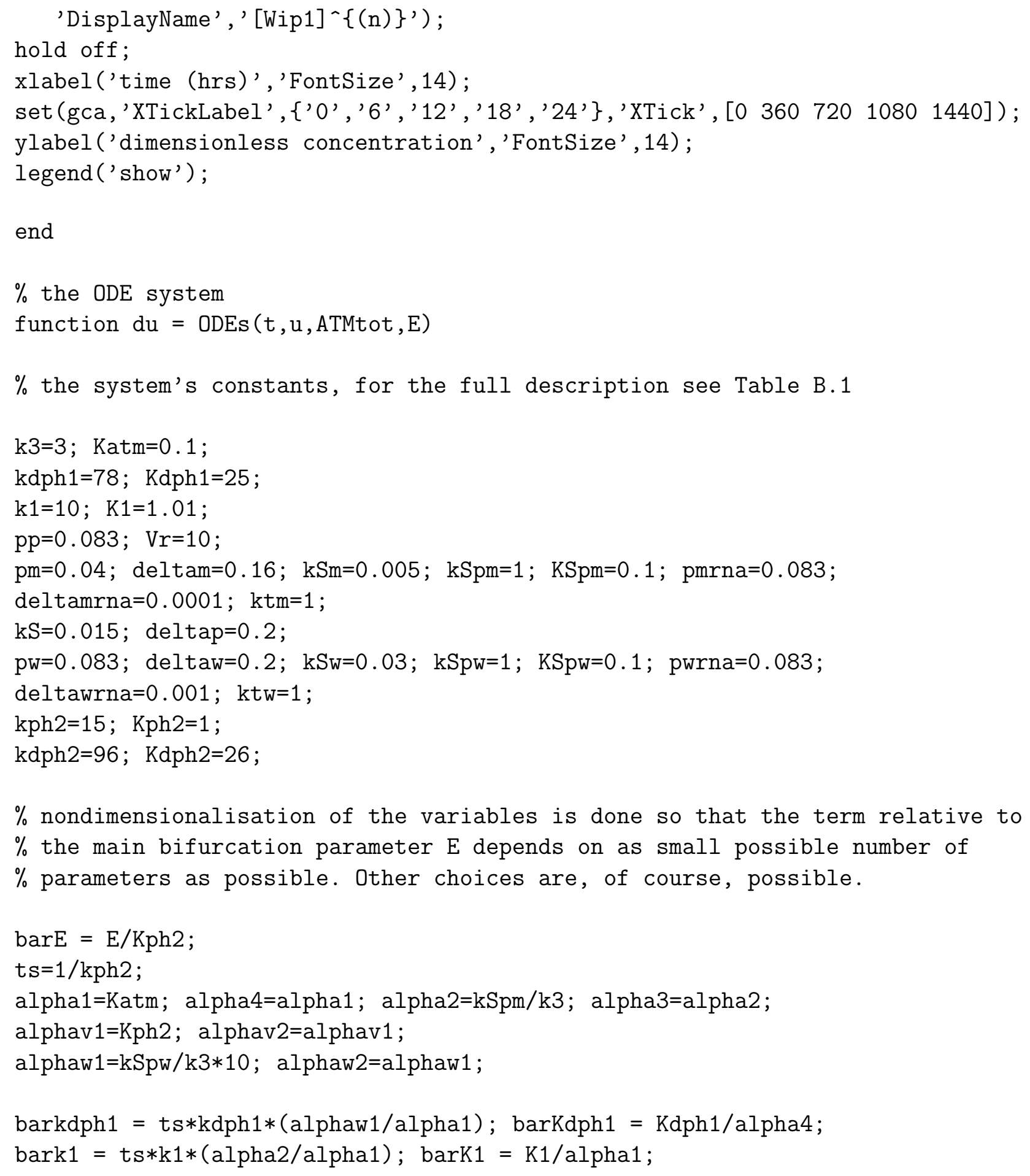


bark3 = ts*k3*(alphav2/alpha1); barKatm = Katm/alpha1;

barpp=ts*pp; barpm=ts*pm; bardeltam=ts*deltam;

barkSm=ts*kSm/alpha3; barkSpm=ts*kSpm/alpha3; barKSpm=KSpm/alpha4;

barpmrna=ts*pmrna; bardeltamrna=ts*deltamrna;

barpw=ts*pw; bardeltaw=ts*deltaw;

barkSw=ts*kSw/alphaw2; barkSpw=ts*kSpw/alphaw2; barKSpw=KSpw/alpha4;

barpwrna=ts*pwrna; bardeltawrna=ts*deltawrna;

barkdph2=ts*kdph2*(alphaw1/alphav2); barKdph2=Kdph2/(alphav2^2);

barkS=ts*kS/alpha1; bardeltap=ts*deltap;

barktm=ts*ktm; barktw=ts*ktw;

ATMtot=ATMtot/alphav1;

$\%$ solve $d u / d t=f(t, u)$

$d u=\operatorname{zeros}(12,1)$;

$\%$ equations for the nucleus (B.1)

$\%$ p53

$\mathrm{du}(1)=\operatorname{barkdph} 1 * u(5) *(\mathrm{u}(4) /(\operatorname{barKdph} 1+\mathrm{u}(4)))-\operatorname{bark} 1 * u(2) *(\mathrm{u}(1) /(\operatorname{barK} 1+u(1))) \ldots$ $-\operatorname{bark} 3 * u(7) *(u(1) /(\operatorname{barKatm}+u(1)))-\operatorname{barpp} * \operatorname{Vr} *(u(1)-u(8))$;

$\% \operatorname{Mdm} 2$

$\mathrm{du}(2)=-\operatorname{barpm} * \operatorname{Vr} *(\mathrm{u}(2)-\mathrm{u}(9))-\operatorname{bardeltam} * u(2)$;

$\%$ Mdm2 mRNA

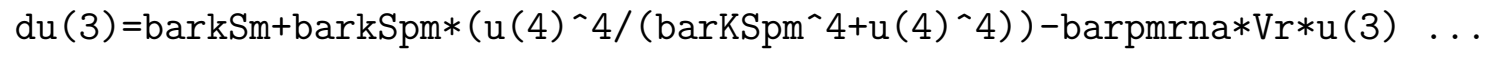
-bardeltamrna*u (3);

$\%$ p53_p

$\mathrm{du}(4)=\operatorname{bark} 3 * u(7) *(u(1) /(\operatorname{barKatm}+u(1)))-\operatorname{barkdph} 1 * u(5) *(u(4) /(\operatorname{barKdph} 1+u(4)))$;

$\%$ Wip1

$\mathrm{du}(5)=\operatorname{barpw} * \operatorname{Vr} * u(11)-\operatorname{bardeltaw} * u(5)$;

$\%$ Wip1 mRNA

$\mathrm{du}(6)=\operatorname{barkSw}+\operatorname{barkSpw} *\left(u(4) \wedge 4 /\left(\operatorname{barKSpw}^{\wedge} 4+u(4) \wedge 4\right)\right)-\operatorname{barpwrna} * \operatorname{Vr} * u(6) \ldots$ -bardeltawrna*u(6);

$\%$ Atm_p

$\mathrm{du}(7)=2 * \operatorname{barE} *(((\operatorname{ATMtot}-\mathrm{u}(7)) / 2) /(1+((\operatorname{ATMtot}-\mathrm{u}(7)) / 2))) \ldots$

$2 * \operatorname{barkdph} 2 * u(5) *\left(u(7)^{\wedge} 2 /\left(\operatorname{barKdph} 2+u(7)^{\wedge} 2\right)\right)$;

$\%$ equations for the cytoplasm (B.2)

$\%$ p53 


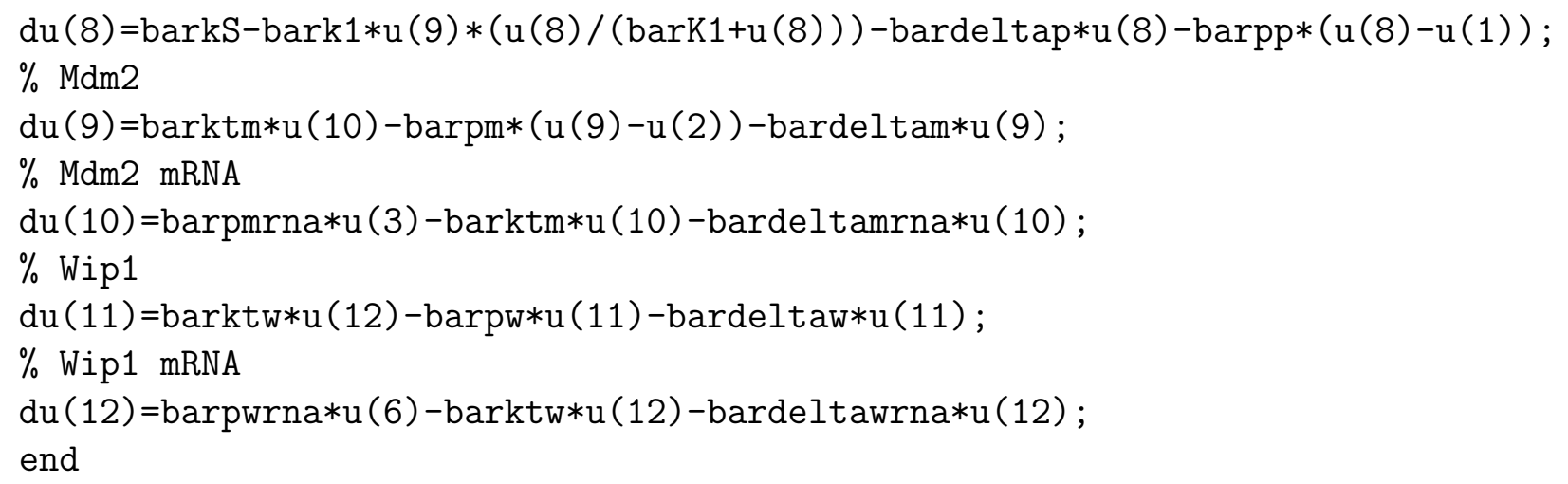




\section{Appendix E. Other figures and plots}

(a)

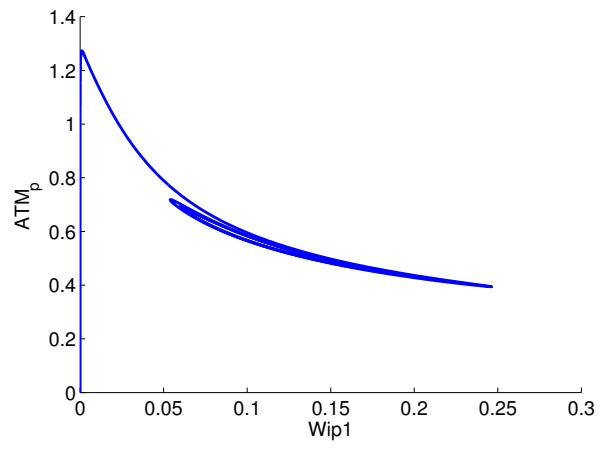

(b)

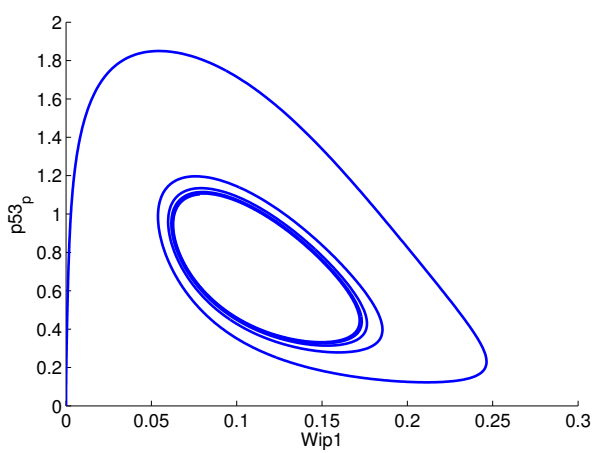

Figure E.1: Phase planes relative to the involved nuclear proteins within $[E]=$ $0.1 \mu M$ (a) Wip1 and $A T M_{p}$ (b) Wip1 and $p 53_{p}$.

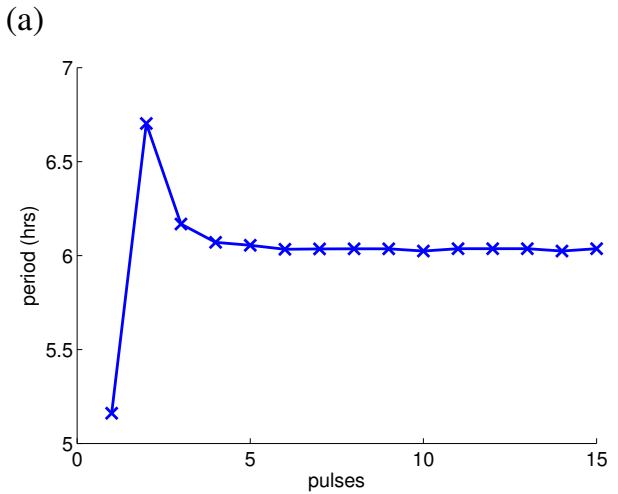

Figure E.2: (a) The periods of the fifteen $p 53_{p}$ pulses produced by the ODE model within $[E]=0.1 \mu M$. The length of the first pulse is 5.16 hours, the $p 53_{p}$ stable limit cycle is of the period $\sim 6$ hours. 
(a)

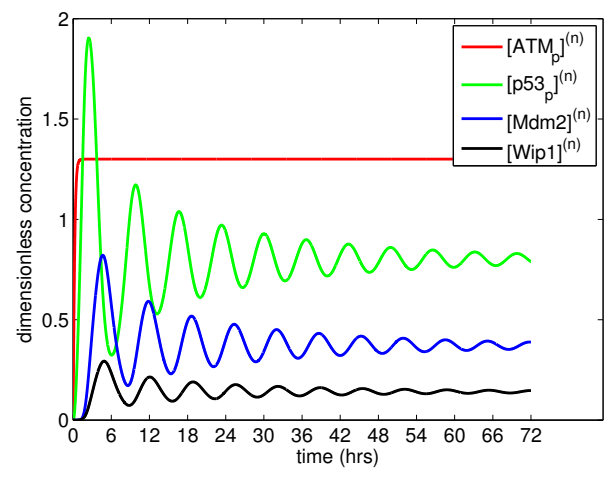

(c)

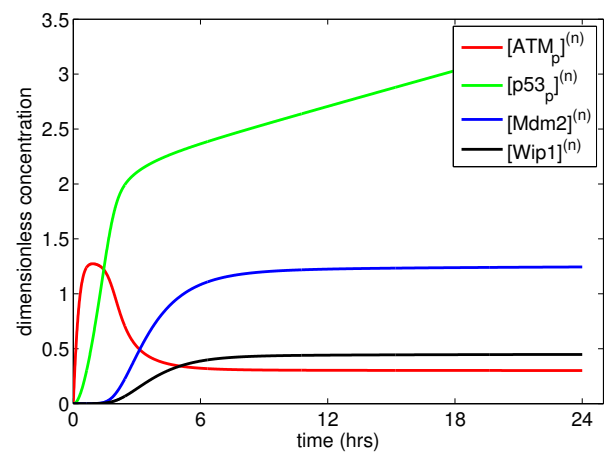

(e)

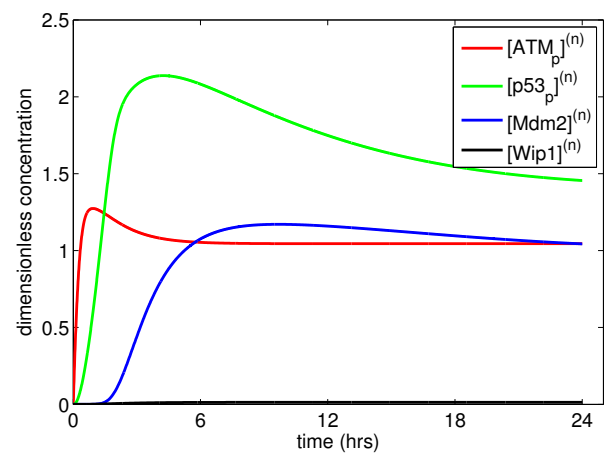

(b)

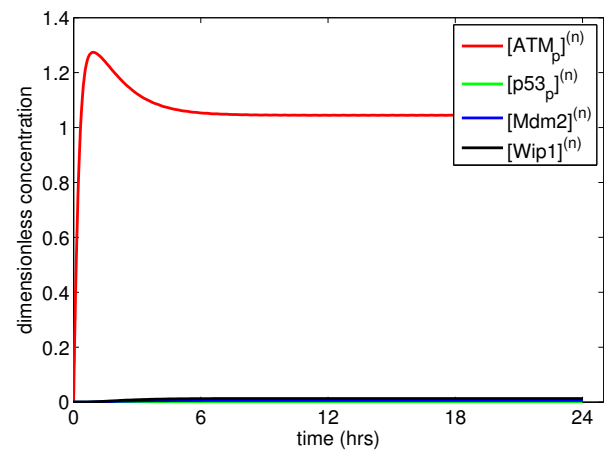

(d)

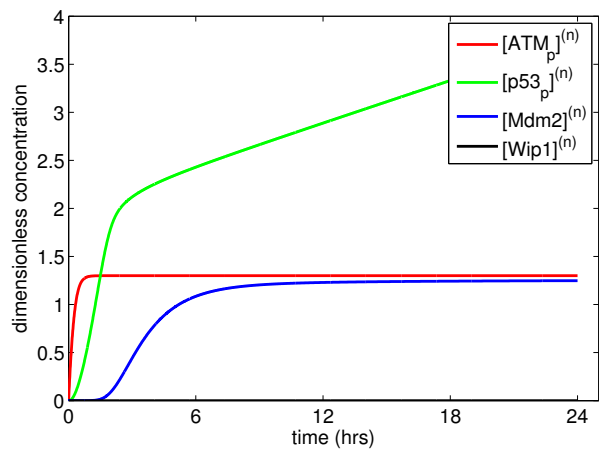

(f)

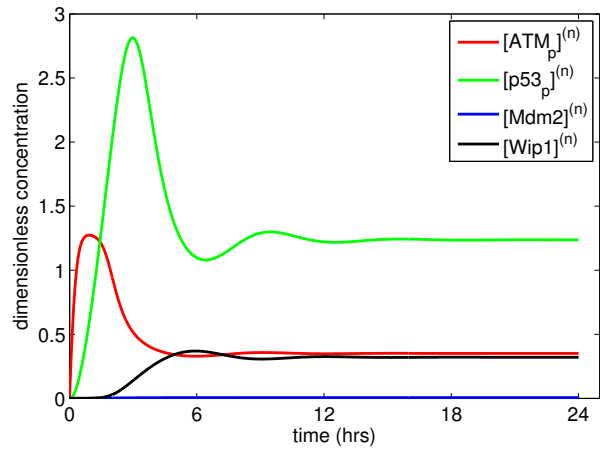

Figure E.3: (a) Inhibition of the negative feedback Wip1 $\dashv A T M, k_{d p h 2}=0$ in (4). (b) Inhibition of the positive feedback $A T M \rightarrow p 53, k_{3}=0$ in (6) (c) Inhibition of the negative feedback Wip $1 \dashv p 53_{p}, k_{d p h 1}=0$ in (6). (d) Inhibition of the positive feedback $p 53_{p} \rightarrow$ Wip $1, k_{S_{p w}}=0$ and $k_{S_{w}}=0$ (the basal production rate). (e) Inhibition of the positive feedback $p 53_{p} \rightarrow$ Wip $1, k_{S_{p w}}=0$ and $k_{S_{w}}=0.03$ (the basal production rate). (f) Inhibition of the positive feedback $p 53_{p} \rightarrow M d m 2$, $k_{S_{p m}}=0$ and $k_{S_{m}}=0.005$ (the basal prodaction rate). 


\section{References}

[1] M. Kracikova, et al., A threshold mechanism mediates p53 cell fate decision between growth arrest and apoptosis, Cell Death \& Differentiation 20 (2013) 576-588.

[2] J. Clairambault, Modelling physiological and pharmacological control on cell proliferation to optimise cancer treatments, Mathematical Modelling of Natural Phenomena 4 (2009) 12-67.

[3] J. Clairambault, O. Fercoq, Physiologically structured cell population dynamic models with applications to combined drug delivery optimisation in oncology, in: M. Bachar, J. Batzel, M. Chaplain (Eds.), Mathematical modelling of cancer growth and treatment, Springer, New York, 2013, Submitted.

[4] F. Murray-Zmijewski, E.A. Slee, X. Lu, A complex barcode underlies the heterogeneous response of p53 to stress, Nature, Rev. Mol. Cell Biol. 9 (2008) 702-712.

[5] L. Dimitrio, Modelling nucleocytoplasmic transport with application to the intracellular dynamics of the tumor suppressor protein $553 \mathrm{Ph} . \mathrm{D}$. thesis, Université Pierre et Marie Curie, Paris, 2012.

[6] L. Dimitrio, J. Clairambault, R. Natalini, A spatial physiological model for p53 intracellular dynamics, Journal of Theoretical Biology 316 (2013) 9-24.

[7] B. Vogelstein, D. Lane, A.J. Levine, Surfing the p53 network, Nature 408 (2000) 307-310.

[8] T.T. Paull, J.H. Lee, The Mre11/Rad50/Nbs1 complex and its role as a DNA double-strand break sensor for ATM, Cell Cycle 4:6 (2005) 737-740.

[9] F. Lévi, A. Okyar, S. Dulong, P.F. Innominato, J. Clairambault, Circadian Timing in Cancer Treatments, Annual Review of Pharmacology and Toxicology $\mathbf{5 0}$ (2010) 377-421.

[10] A. Ballesta, S. Dulong, C. Abbara, B. Cohen, A. Okyar, J. Clairambault, F. Lévi, A Combined Experimental and Mathematical Approach for Molecularbased Optimization of Irinotecan Circadian Delivery, PLoS Comput Biol 7(9): e1002143 (2011) doi:10.1371/journal.pcbi.1002143. 
[11] A. Ballesta, J. Clairambault, S. Dulong, F. Lévi, A Systems Biomedicine Approach for Chronotherapeutics Optimization: Focus on the Anticancer Drug Irinotecan, in: A. d'Onofrio, P. Cerrai, A. Gandolfi (Eds.), New Challenges for Cancer Systems Biomedicine, Part V, SIMAI Lecture Notes, Springer, New York, 2012, 301-327.

[12] F. Billy, J. Clairambault, Designing proliferating cell population models with functional targets for control by anti-cancer drugs, DCDS-B 18 (2013) 865889.

[13] Y. Sun, et al., Treatment-induced damage to the tumor microenvironment promotes prostate cancer therapy resistance through WNT16B, Nature Medicine 18 (2012) 1359-1368.

[14] E. Batchelor, et al., Recurrent Initiation: A mechanism for triggering p53 pulses in response to DNA damage, Molecular Cell 30 (2008) 277-289.

[15] S. Shreeram, et al., Wip1 Phosphatase Modulates ATM-Dependent Signaling Pathways, Molecular Cell 23 (2006) 757-764.

[16] S. Shreeram, et al., Regulation of ATM/p53-dependent suppression of mycinduced lymphomas by Wip1 phosphatase, The Journal of Experimental Medicine 203 (2006) 2793-2799.

[17] G. Lahav, et al., Dynamics of the p53-Mdm2 feedback loop in individual cells, Nature Genetics 36 (2004) 147-150.

[18] N. Geva-Zatorsky, et al., Oscillations and variability in the p53 system, Molecular Systems Biology 2 (2006) 1-13.

[19] R. Lev Bar-Or, et al., Generation of Oscillation by the p53-Mdm2 feedback loop: a theoretical and experimental study, Proc. Natl. Acad. Sci. USA 97 (2000) 11250-11255.

[20] D.R. Green, G. Kroemer, Cytoplasmic functions of the tumour suppressor p53, Nature 458 (2009) 1127-1130.

[21] M.F. Lavin, Ataxia-telangiectasia: from a rare disorder to a paradigm for cell signalling and cancer, Nature 9 (2008) 759-769. 
[22] D.B. Young, et al., Identification of Domains of Ataxia-telangiectasia Mutated Required for Nuclear Localization and Chromatin Association, The Journal of Biological Chemistry 280 (2005) 27587-27594.

[23] M. Fiscella, et al., Wip1, a novel human protein phosphatase that is induced in response to ionizing radiation in a 553 -dependent manner, Proc. Natl. Acad. Sci. USA 94 (1997) 6048-6053.

[24] J.Ch. Bakkenist, M.B. Kastan, DNA damage activates ATM through intermolecular autophosphorylation and dimer dissociation, Nature 421 (2003) 499-506.

[25] M.B. Kastan, J. Bartek, Cell-cycle checkpoints and cancer, Nature 432 (2004) 316-323.

[26] J. Bartkova, et al., ATM activation in normal human tissues and testicular cancer, Cell Cycle 4 (2005) 838-845.

[27] R. Kitagawa, J.Ch. Bakkenist, P.J. McKinnon, M.B. Kastan, Phosphorylation of SMC1 is a critical downstream event in the ATM-NBS1-BRCA1 pathway, Genes \& Development 18 (2004) 1423-1438.

[28] J. Falck, J. Coates, S.P. Jackson, Conserved modes of recruitment of ATM, ATR and DNA-PKcs to sites of DNA damage, Nature 434 (2005) 605-6011.

[29] S.V. Kozlov, et al., Involvement of novel autophosphorylation sites in ATM activation, The EMBO Journal 25 (2006) 3504-3514.

[30] S.V. Kozlov, et al., Autophosphorylation and ATM Activation: additional sites add to the complexity, Journal of Biological Chemistry 286 (2011) 9107 9119.

[31] Y. Sun, et al., DNA Damage-Induced Acetylation of Lysine 3016 of ATM Activates ATM Kinase Activity, Molecular and Cellular Biology 27 (2007) 8502-8509.

[32] E. Berkovich, R.J. Monnat Jr., M.B. Kastan, Roles of ATM and NBS1 in chromatin structure modulation and DNA double-strand break repair, Nature Cell Biology 9 (2007) 683-690.

[33] A. Ali, et al., Requirement of protein phosphatase 5 in DNA-damageinduced ATM activation, Genes and Development 18 (2004) 249-254. 
[34] A.A. Goodarzi, et al., Autophosphorylation of ataxia-telangiectasia mutated is regulated by protein phosphatase 2A, The EMBO Journal 23 (2004) 44514461 .

[35] J.P. Keener, J. Sneyd, Mathematical Physiology I: Cellular Physiology, second ed., Springer, 2009.

[36] L.A. Segel, M. Slemrod, The Quasi-Steady-State Assumption: a case study in perturbation, SIAM Review 31:3 (1989) 446-477.

[37] Y. Haupt, R. Maya, A. Kazaz, M. Oren, Mdm2 promotes the rapid degradation of p53, Nature 387 (1997) 296-299.

[38] N.D. Marchenko, et al., Stress-mediated nuclear stabilization of p53 is regulated by ubiquitination and importin- $\alpha 3$ binding, Cell Death and Differentiation 17 (2010) 255-267.

[39] R.L. Weinberg, D.B. Veprintsev, A.R. Fersht, Cooperative binding of tetrameric p53 to DNA, J. Mol. Biol 341 (2004) 1145-1159.

[40] L. Ma, et al., A plausible model for the digital response of p53 to DNA damage, Proc. Natl. Acad. Sci. USA 102 (2005) 14266-14271.

[41] J. Wagner, et al., p53-Mdm2 loop controlled by a balance of its feedback strength and effective dampening using ATM and delayed feedback, Proc. Natl. Acad. Sci. USA 102 (2005) 109-118.

[42] A. Ciliberto, B. Novak, J.J. Tyson, Steady states and oscillations in the p53/Mdm2 network, Cell Cycle 4 (2005) 488-493.

[43] T. Zhang, P. Brazhnik, J.J. Tyson, Exploring mechanisms of the DNAdamage response: p53 pulses and their possible relevance to apoptosis, Cell Cycle 6 (2007) 85-94.

[44] X.-P. Zhang, F. Liu, W. Wang, Two-phase dynamics of p53 in the DNA damage response, Proc. Natl. Acad. Sci. USA 22 (2011) 8990-8995.

[45] J.K. Kim, T.L. Jackson, Mechanisms That Enhance Sustainability of p53 Pulses, PLoS 8(6) (2013) e65242. doi:10.1371/journal.pone.0065242.

[46] K. Puszyński, B. Hat, T. Lipniacki, Oscillations and bistability in the stochastic model of p53 regulation, J. Theor. Biol. 254 (2008) 452-465. 
[47] M. Sturrock, A.J. Terry, D.P. Xirodimas, A.M. Thompson, M.A.J. Chaplain, Spatio-temporal modelling of the Hes1 and p53-Mdm2 intracellular signalling pathways, Journal of Theoretical Biology 273 (2011) 15-31.

[48] M. Sturrock, A.J. Terry, D.P. Xirodimas, A.M. Thompson, M.A.J. Chaplain, Influence of the Nuclear Membrane, Active Transport, and Cell Shape on the Hes1 and p53Mdm2 Pathways: Insights from Spatio-temporal Modelling, Bulletin of Mathematical Biology 74 (2012) 1531-1579.

[49] M. Sturrock, A. Hellander, A. Matzavoinos, M.A.J. Chaplain, Spatial stochastic modelling of the Hes1 gene regulatory network: intrinsic noise can explain heterogeneity in embryonic stem cell differentiation, J. R. Soc. Interface 10, 20120988 (2013) 9 pages.

[50] A. Dhooge, W. Govaerts, Y.A. Kuznetsov, Matcont: A matlab package for numerical bifurcation analysis of ODEs, ACM TOMS 29 (2003) 141-164.

[51] Y.A. Kuznetsov, Elements of Applied Bifurcation Theory, third ed., Springer-Verlag, 2004.

[52] L. Dimitrio, J. Clairambault, J.P. Françoise, Bifurcation in the p53 system: a compartmental approach (2013), in preparation.

[53] Y. Lee, M.J. Chong, P.J. McKinnon, Ataxia Telangiectasia Mutateddependent apoptosis after genotoxic stress in the developing nervous system is determined by cellular differentiation status, The J. of Neuroscience 21 (2001) 6687-6693.

[54] J.Ch. Bakkenist, M.B. Kastan, Initiating Cellular Stress Responses, Cell 118 (2004) 9-17.

[55] J.H. Lee, T.T. Paull, ATM activation by DNA double-strand breaks through the Mre11-Rad50-Nbs1 complex, Science 308 (2005) 551-554.

[56] M. Sturrock, A. Hellander, S. Aldakheel, L. Petzold, M.A.J. Chaplain, The role of dimerisation and nuclear transport in the Hes1 gene regulatory network Bull. Math. Biol. DOI 10.1007/s11538-013-9842-5 (2013), Accepted. 